This is the final peer-reviewed accepted manuscript of

Franchi F; Hofmann A; CAVALAZZI, BARBARA; Wilson A; BARBIERI, ROBERTO: DIFFERENTIATING MARINE VS HYDROTHERMAL PROCESSES IN DEVONIAN CARBONATE MOUNDS USING RARE EARTH ELEMENTS (KESS KESS MOUNDS, ANTI-ATLAS, MOROCCO). CHEMICAL GEOLOGY, 409. ISSN 0009-2541

DOI: $10.1016 /$ j.chemgeo.2015.05.006

The final published version is available online at:

http://dx.doi.org/10.1016/j.chemgeo.2015.05.006

Rights / License:

The terms and conditions for the reuse of this version of the manuscript are specified in the publishing policy. For all terms of use and more information see the publisher's website.

This item was downloaded from IRIS Università di Bologna (https://cris.unibo.it/)

When citing, please refer to the published version. 


\title{
Differentiating marine vs hydrothermal processes in Devonian carbonate mounds using rare earth elements (Kess Kess mounds, Anti-Atlas, Morocco)
}

\author{
Fulvio Franchi ${ }^{\text {a,b,c,* }}$, Axel Hofmann ${ }^{\mathrm{d}}$, Barbara Cavalazzi ${ }^{\mathrm{c}, \mathrm{d}}$, Allan Wilson $^{\mathrm{e}}$, Roberto Barbieri ${ }^{\mathrm{c}}$ \\ a Department of Earth and Environmental Sciences, BIUST, Private Mail Bag 16, Plot 10071, Palapye, Botswana \\ b ISMAR, CNR, Bologna, Via Gobetti 101, 40129 Bologna, Italy \\ ' Dipartimento di Scienze Biologiche, Geologiche e Ambientali, Università di Bologna, Via Zamboni 67, 40126 Bologna, Italy \\ d Department of Geology, University of Johannesburg, PO Box 524, Auckland Park 2006, Johannesburg, South Africa \\ e School of Geosciences, University of Witwatersrand, 1 Jan Smuts Avenue, Braamfontein 2000, Johannesburg, South Africa
}

A R T I C L E I N F O

\section{Keywords:}

\section{Kess Kess}

Carbonate mounds

Hydrothermalism

Carbonate

geochemistry Rare

earth elements Palaeo-

redox

\begin{abstract}
A B S T R A C T
Petrological and geochemical analyses were carried out on Early Devonian Kess Kess mound limestones of the Seheb el Rhassel Group exposed in the Hamar Laghdad Ridge (Tafilalt Platform, Eastern Anti Atlas, Morocco) in order to evaluate marine vs hydrothermal processes for the origin of the mounds. Hydrothermal fluid circulation affected limestone deposition resulting in the formation of a plumbing system preserved in the mound facies and inter mound facies as cavities, veins, and dykes. Shale normalized rare earth element (REE) patterns for limestones display pronounced light REE depletion compared to middle REE (average $\operatorname{Pr}_{\mathrm{SN}} / \mathrm{Dy}_{\mathrm{SN}}=0.46$ ) and heavy REE (average $\operatorname{Pr}_{\mathrm{SN}} / \mathrm{Yb}_{\mathrm{SN}}=0.55$ ), a super chondritic Y/Ho ratio (up to 87) and positive La anomaly consistent with precipitation from normal marine seawater. With in our data set the Ce anomaly, as well as $U$ and other trace element concentrations vary with facies providing evidence for variable redox conditions. The presence of positive Ce anomaly in carbonates of the plumbing system is consistent with precipitation under anoxic condition. Samples from mound facies and quartz veinlets in the upper part of the group show REE patterns consistent with precipitation from hydrothermal fluids. Early Devonian hypothetical seawater REE patterns were calculated from samples showing normal marine REE patterns (i.e. inter mound facies) and from samples with weaker marine signature (i.e. fossiliferous mound limestones and plumbing system deposits). Hypothetical patterns are slightly enriched in $\Sigma$ REE compared to modern open ocean seawater. This study reveals that REE analysis is a powerful tool for understanding polygenetic carbonate systems. It sheds light into the genesis of the Kess Kess mounds by reconstructing fluid pathways and palaeo redox conditions.
\end{abstract}

\section{Introduction}

The chemistry of rare earth elements (REE) is a valuable tool to in vestigate marine sedimentary palaeoenvironments. The relative abundance of REE in marine precipitates reflects i) the source of the different fluids in the basin that mix to form seawater (e.g. Kamber et al., 2004; Feng et al., 2009), and ii) variations in alkalinity and oxygen levels (Elderfield, 1988; Piepgras and Jacobsen, 1992; Bertram and Elderfield, 1993; Greaves et al., 1999; Kim et al., 2012; Hu et al., 2014). The REE pattern of marine precipitates can be considered in terms of marine, terrestrial, and hydrothermal fluid endmembers. The REEcomposition of carbonates as a proxy for seawater composition

* Corresponding author at: Department of Earth and Environmental Sciences, BIUST, Private Mail Bag 16, Plot 10071, Palapye, Botswana. Tel.: + 26774531017.

E-mail address: franchif@biust.ac.bw (F. Franchi). and palaeoenvironmental and palaeoecological reconstructions has been applied for Precambrian (Kamber and Webb, 2001; Van Kranendonk et al., 2003; Allwood et al., 2010; and references therein) and Phanerozoic (Kawabe et al., 1991; Webb and Kamber, 2000; Tanaka et al., 2003; Nothdurft et al., 2004; Shields and Webb, 2004; Johannesson et al., 2006; Olivier and Boyet, 2006; Oliveri et al., 2010) strata. Particularly it was demonstrated that Devonian reef carbonates (Canning Basin, Western Australia) retained consistent seawater like REE signatures (Nothdurft et al., 2004), suggesting the usefulness of an cient carbonate REE patterns as a seawater proxy. The enrichment of certain trace elements (e.g. U, Mo, etc.) and behavior of redox sensitive REE (Eu, Ce) have been used to investigate recent (e.g. Hu et al., 2014) and ancient (e.g. Kamber and Webb, 2001) carbonates giving new in sights into the redox conditions during early diagenesis. The aim of the present study is to explore the Kess Kess carbonate mounds by using REE and other trace elements. 
The Early Devonian (Emsian) Kess Kess mounds are well exposed at the Hamar Laghdad Ridge in the Tafilalt region (Eastern Anti Atlas, Morocco; Figs. 12 2). A hydrothermal origin, based on temperature estimates from fluid inclusions, REE composition, and oxygen isotopic composition of carbonates, and the presence of putative thermophilic faunas was proposed to explain the formation of these micritic mounds (Bełka, 1998; Mounji et al., 1998; Aitken et al., 2002; Berkowski, 2004,
2006, 2008; Bełka and Berkowski, 2005; Olempska and Bełka, 2010; Berkowski and Klug, 2012; Jakubowicz et al., 2013; Franchi et al., 2014). Warm fluids ( $3156^{\circ} \mathrm{C}$, Mounji et al., $1998 ; 40 \quad 45^{\circ} \mathrm{C}$ Franchi et al., 2014) were probably derived from low temperature hydrother mal activity driven by late stage magmatic activity related to Early De vonian submarine calc alkaline volcanism. Recently, evidence of this hydrothermal activity was reported from the upper part of the Hamar

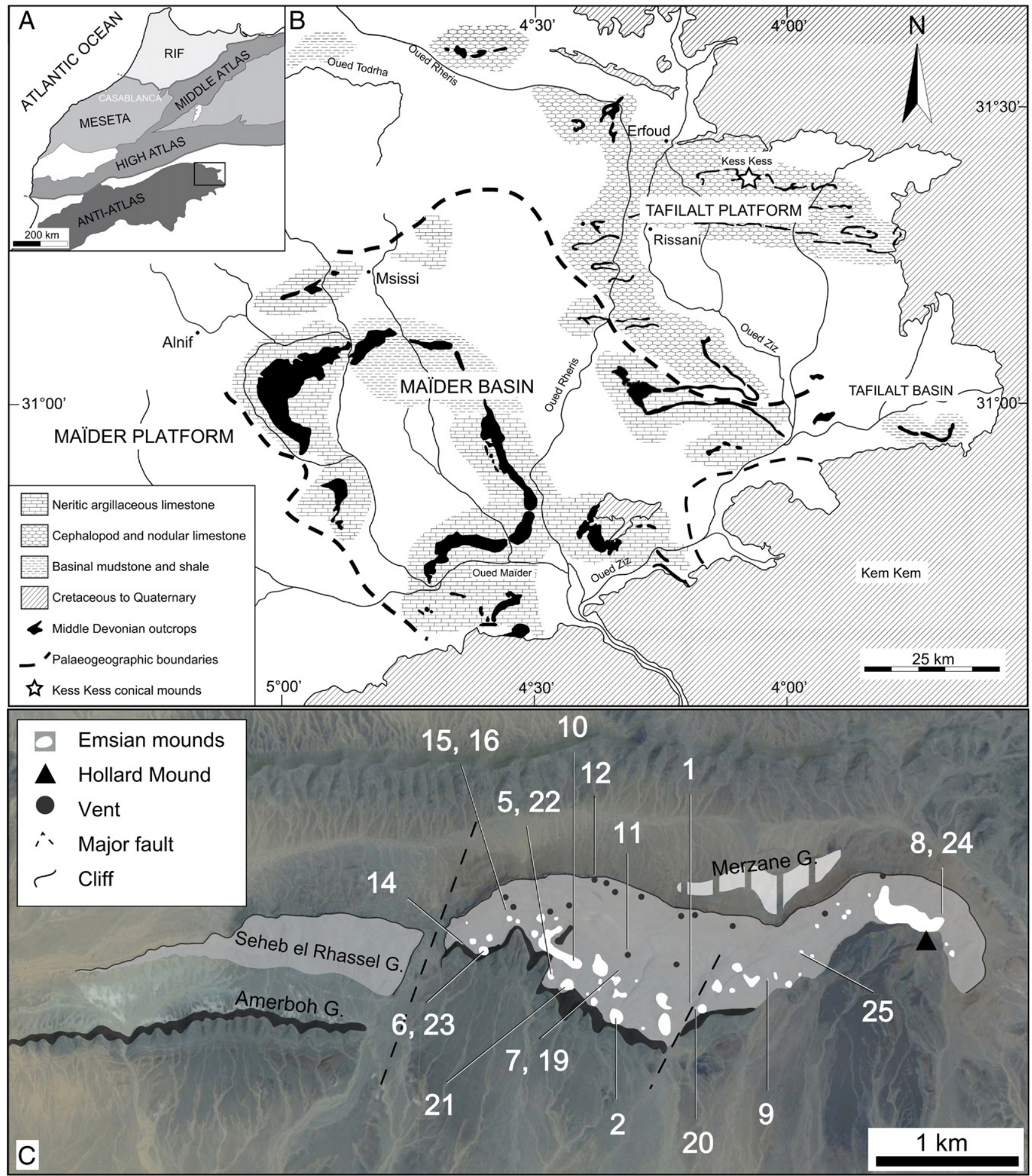

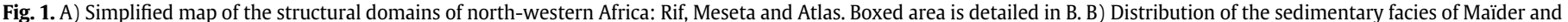

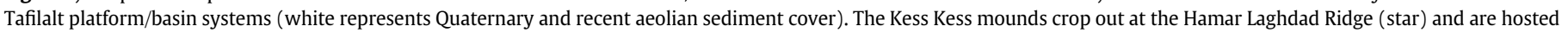

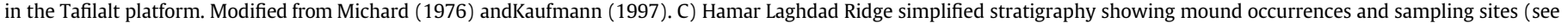
Table 1). 


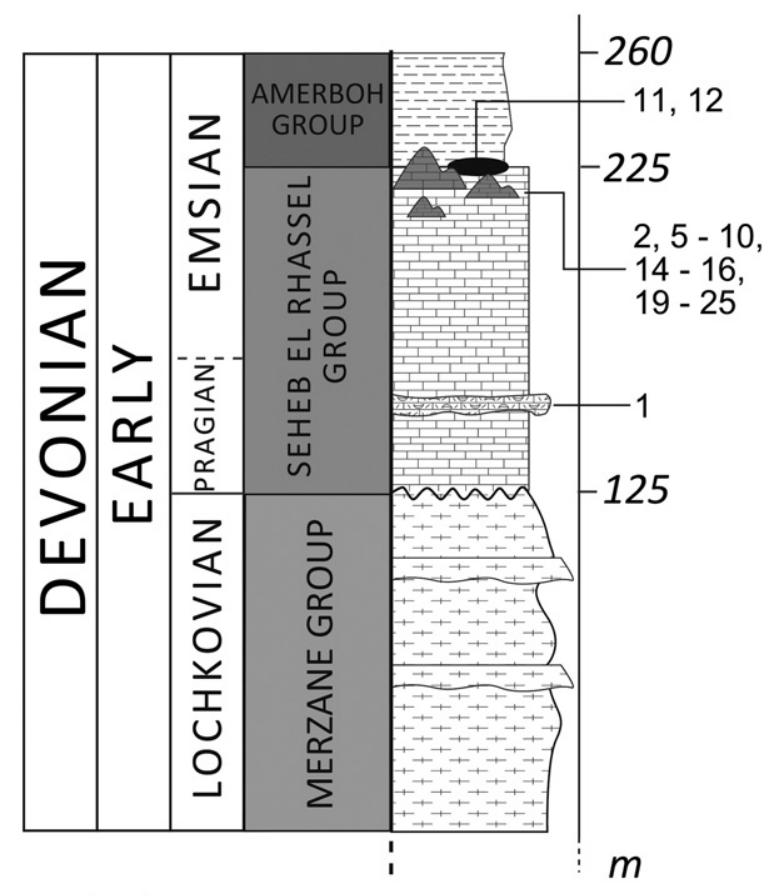

Volcanic breccia

\section{Limestone}

\section{Coquina}

\section{Marl}

\section{Erosive surface}

\section{Mound}

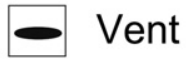

Fig. 2. Lithostratigraphic subdivision of the Hamar Laghdad Ridge showing the stratigraphic position (smaller numbers) of the studied samples.

Laghdad limestone succession (Seheb el Rhassel Group) where components of an articulated plumbing system of veins, dykes, and vents were described (Franchi et al., 2014). However, the hydrothermal origin of the Kess Kess mounds is still debated because the oxygen isotopic com position $\left(\delta^{18} \mathrm{O}\right.$ values as low as $-11 \%$ PDB; Franchi et al., 2014) of the Early Devonian limestones is inconclusive and considered inconsistent with unambiguous hydrothermal genesis and probably due to incipient burial diagenesis (Joachimski et al., 1999; Klug et al., 2009).

Studies of the REE distribution of Kess Kess carbonate may shed light on this issue, as REE in carbonate tend to be relatively immobile during diagenesis (Banner et al., 1988; Webb and Kamber, 2000; Nothdurft et al., 2004), and REE behavior in aqueous solution is redox sensitive. A limited REE dataset from the Hamar Laghdad Ridge was previously re ported by Bełka (1998), who compared mound limestone signatures with those from neptunian dykes and intermound deposits, suggesting a relation between warm fluid circulation and REE pattern. However, Bełka (1998) provided results from only five samples, three of which were obtained from neptunian dykes which were considered to be of younger age (Frasnian; e.g. Peckmann et al., 1999). These fractures are probably related to development of younger mounds (i.e. Hollard Mound; Fig. 1C) generated by hydrocarbon seepage (e.g. Peckmann et al., 1999, 2005; Cavalazzi et al., 2007). In order to avoid comparative studies of unrelated facies, more work is thus required. Here we present a broader data set from different mound and intermound facies, using REE and other trace elements, to elucidate the palaeoenvironmental conditions in which the Kess Kess mounds formed.

\section{Geological setting and sample description}

Palaeozoic deposits in the eastern Anti Atlas of Morocco are part of the continental shelf sequence that developed along the north western margin of Gondwana during Late Precambrian to Early Carbon iferous (Piqué and Michard, 1989). The palaeolatitude of these deposits during the Early Devonian has tentatively been placed between $35^{\circ} \mathrm{S}$ (Mounji et al., 1998) and $60^{\circ} 70^{\circ} \mathrm{S}$ (Tait et al., 1995; Stampfli and Borel, 2002) on the southern margin of the Palaeo Tethys. The closure of the western Palaeo Tethys and the consequent clockwise rotation of Gondwana during the Devonian resulted in a gradual formation, along the northern continental margin of the West African Craton, of several intra cratonic basins (Wendt, 1985) that include the Tafilalt platform/ basin system (Fig. 1).

The Hamar Laghdad Ridge consists of a W E oriented succession of Early to Middle Devonian sedimentary rocks considered as a part of the Tafilalt Platform (Figs. 12 ). During the Lochkovian, the deposition of volcanic rocks of the Merzane Group (Hollard, 1981) in a shallow epicontinental sea led to the formation of a topographic high that became the depositional site of the Seheb el Rhassel Group (SRG) limestones during the Pragian Emsian (Aitken et al., 2002). This group consists of ca. $100 \mathrm{~m}$ of fossiliferous limestones (Fig. 2) composed of mottled micrite/microsparite with variable amounts of skeletal debris and carbonate cements. The bottom strata of the SRG are made of brachiopod and crinoid packstone/rudstone. The Kess Kess mounds lie on the top of the SRG(Fig. 2) and are covered by the argillaceous deposits of the Amerboh Group (Aitken et al., 2002; Berkowski, 2008; Berkowski and Klug, 2012). The mounds developed in relatively deep water below fair weather wave base, probably below the photic zone (Bełka, 1998; Aitken et al., 2002; Berkowski, 2008, 2012) between 200 and 300 meters according to Mounji et al. (1998). Decimeter wide neptunian dykes, interpreted as syndepositional fractures (see Cavalazzi et al., 2007), cross cut the whole succession from the Merzane volcanic rocks to the boundary between SRG and Amerboh Group (Fig. 2).

Sampling sites for this study were carefully selected on the basis of previous field studies and petrographic and mineralogical data (Cavalazzi et al., 2007; Franchi et al., 2014). Samples for this work were collected from different facies of the SRG corresponding to differ entdepositional settings (Figs. 1C, 2 and 3). Among 20 studied samples, one sample is a brachiopod coquina collected from the middle part of the group, 17 are limestones from mound and inter mound facies, and two samples are ironstone and quartz bearing rock, respectively, collected from the upper part of the group (Fig. 2).

Brachiopod coquina (sample 1; Table 1) forms meter thick beds or lenses that alternate with crinoidal packstone grainstone. Two generations of syntaxial and scalenohedral calcite form overgrowths on brachiopod shells (Fig. 4A), whereas micritic cements are less than $50 \%$.

Limestone samples from mound buildups (Table 1) are dominated by wackestone (sample 10). Stromatactis bearing fossiliferous limestone represents the bulk of the mound facies (Figs. 3B and 4B). Stromatactis infill consists of several generations of isopachous, radiaxial, fibrous calcite (sample 2; Table 1) and blocky calcite distributed toward the center of the cavities (Fig. 4B, C). The fossiliferous limestones are locally transected by a system of veins regarded hydrothermal in origin. These veins are filled by black and white gener ations of isopachous and botryoidal calcite cements (samples 21, 25; Table 1). Mound facies is locally typified by cavities that were probably part of the plumbing system. These cavities are filled by trilobite bearing limestone with two different fabrics (Figs. $3 \mathrm{C}$ and 4D F): the upper part of cavity fills consists of a cement dominated rudstone 


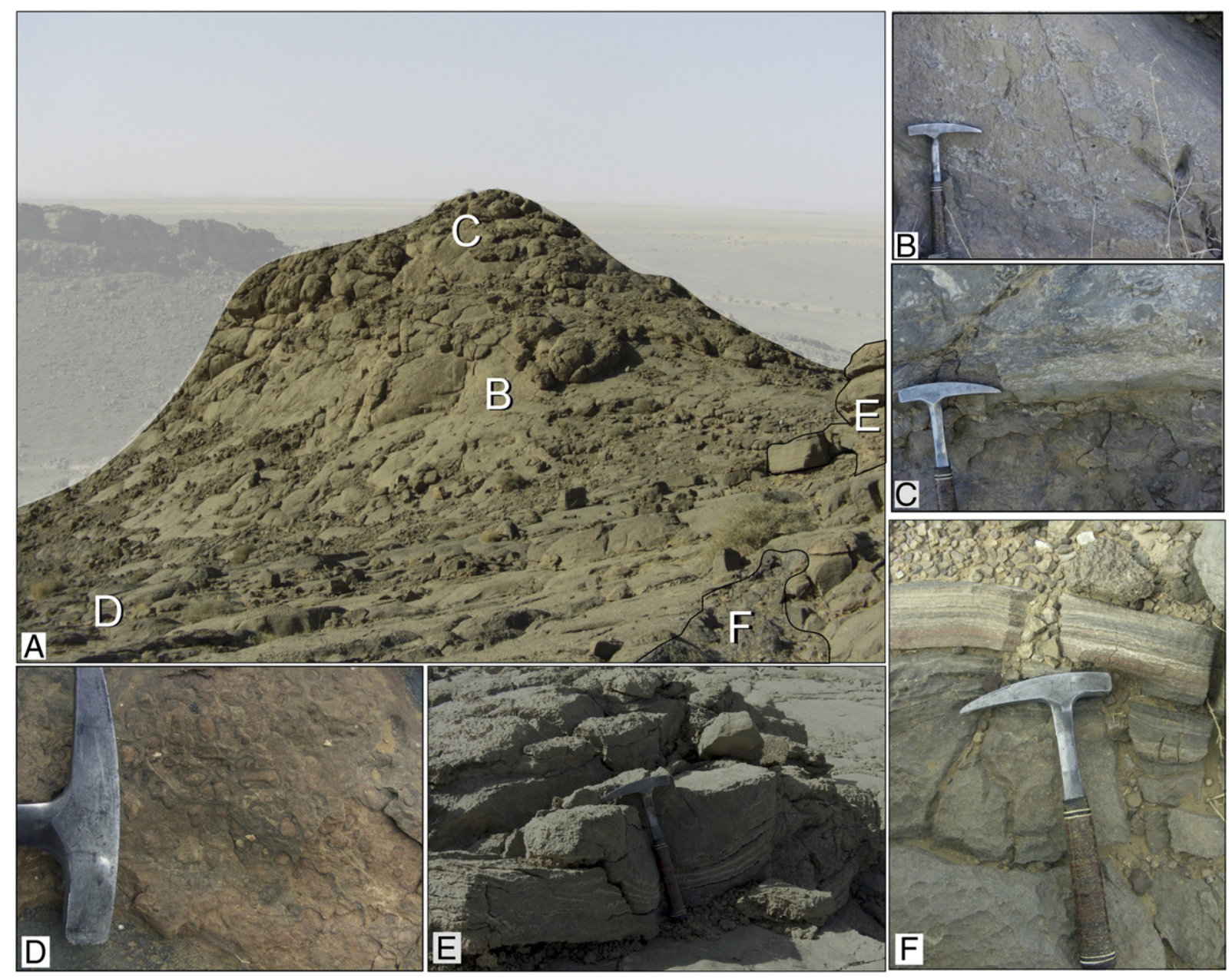

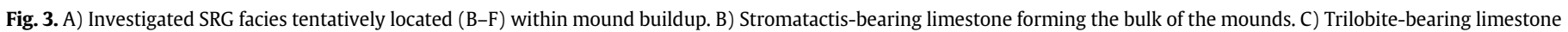

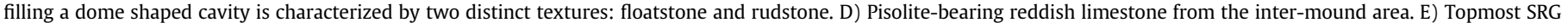
strata composed of highly fractured tentaculite packstone. F) Syndepositional fracture filled with several generations of white, gray and reddish laminated carbonate cements.

(samples 6, 23; Table 1) and the lower part consists of floatstone (samples 5, 22; Table 1). Warm fluid probably circulated through these cavities triggering micrite dissolution and cement precipitation. Mound facies is also typified by layered limestone made of alternations of gray and black micrite and microsparite (samples 8, 24; Table 1).

Inter mound facies interfingers with mound facies in the upper part of the SRG (Berkowski, 2004) and is mainly composed of crinoid trilobite wackestone (Aitken et al., 2002). In the upper part of the SRG the inter mound strata are interbedded with centimeters to decimeters thick reddish clastic layers (samples 9, 14 16, 19; Table 1 ) typified by pisolites, $\mathrm{cm}$ sized carbonate pebbles, and a high percentage of well rounded clasts and pebbles of quartz (Figs. 3D and 4G). This facies, described for the first time by Franchi et al. (2014), has probably been generated by meteoric alteration during subaerial exposure of the Hamar Laghdad succession. One sample (sample 20; Table 1) was collected from the uppermost strata of SRG. These strata consist of discontinuous tentaculite packstone that locally drapes the mounds (Fig. 3E). One sample (sample 7; Table 1) from a fracture which cross cut the SRG inter mound area (Fig. 3F) was collected. The fractures, formerly named neptunian dykes (Cavalazzi et al., 2007), are filled by carbonate debris of Frasnian age (e.g. Peckmann et al., 1999) cemented by several generations of calcite cement and euhedral dolomite (sample 7; Fig. 4H).

Two samples were collected from deposits of ironstone in the upper part of the SRG. These deposits are dominated by globular and spongy masses of primary blackish brown goethite and have been inferred to correspond to fossil vents (Franchi et al., 2014). The ironstone (sample 12; Table 1) is hosted within Fe rich carbonate cemented by zoned, euhedral dolomite locally breached by veins filled with quartz (sample 11; Table 1).

\section{Methods}

A total of twenty samples from different facies of the SRG were analyzed for mineralogical and chemical composition (Tables 13 ). Polished slabs and standard petrographic thin sections were prepared at the University of Bologna. The microfacies were studied with an optical microscope equipped with a Pyser SGI micrometer and a Nikon Coolpix 4500 digital camera. Five samples (Table 3) were analyzed in situ with a WITec alpha300R Confocal Raman microscope at the University of Johannesburg to identify the distribution of mineral phases and the presence of carbonaceous matter within the samples. Raman analyses were recorded and treated using WITecProject2.06® software, and compared with reference spectra from the RRUFFData Base and literature (White, 2009; Cavalazzi et al., 2012 and references therein) using the Crystal Sleuth software (Downs, 2006).

Morphological information and chemical composition of all the samples were obtained using a Jeol 5600 Scanning Electron Microscope (SEM) equipped with an electron back scatteringCENTAURUS system (EDS) at University of Johannesburg. The SEM EDS investigations were performed on etched (60 $\mathrm{s}$ in aqueous solutions with $1 \% \mathrm{HCl}$ concentration) and non etched freshly broken samples, thin sections 
Table 1

Summary of SRG samples. Samples processed for ICP-MS are in gray.

\begin{tabular}{|c|c|c|c|c|c|c|}
\hline Sample & Location & Description & Composition* & Facies & Analyses & Coordinates \\
\hline 1 & inter-mound & $\begin{array}{l}\text { Articulated brachiopod grainstone } \\
\text { (coquina) }\end{array}$ & Calcite & Brachiopod coquina & $\begin{array}{l}\text { XRD, XRF, } \\
\text { EMPA, ICP-MS }\end{array}$ & $\begin{array}{l}31^{\circ} 22^{\prime} 33.98 " \mathrm{~N} \\
4^{\circ} 3 \text { ' } 8.20^{\prime \prime} \mathrm{W}\end{array}$ \\
\hline 2 & mound & $\begin{array}{l}\text { Stromatactis filling - Micrite and } \\
\text { fibrous radiaxial calcite }\end{array}$ & Calcite & Stromatactis & $\begin{array}{l}\text { XRD, XRF, } \\
\text { EMPA, ICP-MS }\end{array}$ & $\begin{array}{l}31^{\circ} 22 ' 27.80^{\prime \prime} \mathrm{N} \\
4^{\circ} 2^{\prime} 58.68^{\prime \prime} \mathrm{W}\end{array}$ \\
\hline 5 & mound & Trilobite floatstone & Calcite, Quartz & Trilobite-bearing limestone & $\begin{array}{l}\text { XRD, XRF, } \\
\text { ICP-MS, RAMAN }\end{array}$ & $\begin{array}{l}31^{\circ} 22^{\prime} 39.61 " \mathrm{~N} \\
4^{\circ} 3 ' 29.02^{\prime \prime} \mathrm{W}\end{array}$ \\
\hline 6 & mound & $\begin{array}{l}\text { Trilobite rudstone with geopetal } \\
\text { micrite }\end{array}$ & Calcite & Trilobite-bearing limestone & $\begin{array}{l}\text { XRD, XRF, EMPA, } \\
\text { ICP-MS,RAMAN }\end{array}$ & $\begin{array}{l}31^{\circ} 22^{\prime} 44.58 " \mathrm{~N} \\
4^{\circ} 3^{\prime} 45.26^{\prime \prime} \mathrm{W}\end{array}$ \\
\hline 7 & inter-mound & $\begin{array}{l}\text { Isopachous generations of fibrous } \\
\text { radiaxial calcite }\end{array}$ & Calcite, Dolomite & Fracture (Neptunian dyke) & $\begin{array}{l}\text { XRD, XRF, } \\
\text { EMPA, ICP-MS }\end{array}$ & $\begin{array}{l}31^{\circ} 22^{\prime} 37.84 " \mathrm{~N} \\
4^{\circ} 3^{\prime} 11.50^{\prime \prime} \mathrm{W}\end{array}$ \\
\hline 8 & mound & $\begin{array}{l}\text { Black and grey micrite interbedded } \\
\text { with blocky and radiaxial calcite }\end{array}$ & Calcite, Quartz & Dark layered micrite & $\begin{array}{l}\text { XRD, XRF, EMPA, } \\
\text { ICP-MS,RAMAN }\end{array}$ & $\begin{array}{l}31^{\circ} 22^{\prime} 53.23 " \mathrm{~N} \\
4^{\circ} 1^{\prime} 49.44^{\prime \prime} \mathrm{W}\end{array}$ \\
\hline 9 & inter-mound & $\begin{array}{l}\text { Reddish quartz-rich micrite with } \\
\text { centimeter sized pisolites }\end{array}$ & Calcite, Quartz & Pisolite-bearing limestone & $\begin{array}{l}\text { XRD, XRF, } \\
\text { EMPA, ICP-MS }\end{array}$ & $\begin{array}{l}31^{\circ} 22^{\prime} 36.54 " \mathrm{~N} \\
4^{\circ} 2^{\prime} 31.50^{\prime \prime} \mathrm{W}\end{array}$ \\
\hline 10 & mound & $\begin{array}{l}\text { Wackestone with prevalent auloporids } \\
\text { and trilobite bioclasts }\end{array}$ & Calcite & Fossiliferous limestone & $\begin{array}{l}\text { XRD, XRF, } \\
\text { EMPA, ICP-MS }\end{array}$ & $\begin{array}{l}31^{\circ} 22^{\prime} 41.97 " \mathrm{~N} \\
4^{\circ} 3^{\prime} 19.39^{\prime \prime} \mathrm{W}\end{array}$ \\
\hline 11 & vent & $\begin{array}{l}\text { Reddish rocks occuring near the vent } \\
\text { structures }\end{array}$ & Quartz, Calcite & Quartz filling veinlets & $\begin{array}{l}\text { XRD, XRF, } \\
\text { EMPA, RAMAN }\end{array}$ & $\begin{array}{l}31^{\circ} 22^{\prime} 40.30^{\prime \prime} \mathrm{N} \\
4^{\circ} 3^{\prime} 15.52^{\prime \prime} \mathrm{W}\end{array}$ \\
\hline 12 & vent & $\begin{array}{l}\text { Vent filling consisting of spongy } \\
\text { ironstone }\end{array}$ & Goethite & Goethite & $\begin{array}{l}\text { XRD, XRF, } \\
\text { EMPA, RAMAN }\end{array}$ & $\begin{array}{l}31^{\circ} 22^{\prime} 53.09 " \mathrm{~N} \\
4^{\circ} 3{ }^{\prime} 29.38^{\prime \prime} \mathrm{W}\end{array}$ \\
\hline 14 & inter-mound & $\begin{array}{l}\text { Reddish layered limestone consisting } \\
\text { of micro and mesosparite }\end{array}$ & Calcite, Goethite(?) & Reddish layered limestone & $\mathrm{XRD}$ & $\begin{array}{l}31^{\circ} 22^{\prime} 46.66^{\prime \prime} \mathrm{N}, \\
4^{\circ} 3^{\prime} 47.89^{\prime \prime} \mathrm{W}\end{array}$ \\
\hline 15 & inter-mound & Reddish dolomitized limestone & Calcite, Dolomite & Dolomitized limestone & XRD & $\begin{array}{l}31^{\circ} 22^{\prime} 52.09 " \mathrm{~N} \\
4^{\circ} 3{ }^{\prime} 38.54^{\prime \prime} \mathrm{W}\end{array}$ \\
\hline 16 & inter-mound & $\begin{array}{l}\text { Reddish dolomitized carbonatic breccia } \\
\text { with rounded pebbles of limestone }\end{array}$ & $\begin{array}{l}\text { Calcite, Dolomite, } \\
\text { Quartz }\end{array}$ & Dolomitized limestone & XRD & $\begin{array}{l}31^{\circ} 22^{\prime} 52.09 " \mathrm{~N} \\
4^{\circ} 3{ }^{\prime} 38.54 " \mathrm{~W}\end{array}$ \\
\hline 19 & inter-mound & $\begin{array}{l}\text { Reddish dolomitized carbonatic breccia } \\
\text { with rounded pebbles of calcite }\end{array}$ & $\begin{array}{l}\text { Calcite, Dolomite, } \\
\text { Quartz }\end{array}$ & Dolomitized limestone & $\mathrm{XRD}$ & $\begin{array}{l}31^{\circ} 22^{\prime} 40.30^{\prime \prime} \mathrm{N} \\
4^{\circ} 3^{\prime} 15.52^{\prime \prime} \mathrm{W}\end{array}$ \\
\hline 20 & inter-mound & $\begin{array}{l}\text { Tentaculite wackestone/packstone } \\
\text { interbedded with radiaxial calcite }\end{array}$ & Calcite, Quartz & Tentaculite packstone & $\begin{array}{l}\text { XRD, XRF, } \\
\text { EMPA, ICP-MS }\end{array}$ & $\begin{array}{l}31^{\circ} 22^{\prime} 31.72 " \mathrm{~N} \\
4^{\circ} 2^{\prime} 48.98^{\prime \prime} \mathrm{W}\end{array}$ \\
\hline 21 & mound & $\begin{array}{l}\text { Isopachous generations of fibrous } \\
\text { radiaxial calcite }\end{array}$ & Calcite & Vein filling & $\begin{array}{l}\text { XRD, XRF, } \\
\text { EMPA, ICP-MS }\end{array}$ & $\begin{array}{c}31^{\circ} 22^{\prime} 36.95^{\prime \prime} \mathrm{N} \\
4^{\circ} 3 \text { '23.46"W }\end{array}$ \\
\hline 22 & mound & $\begin{array}{l}\text { Reddish layered micrite with trilobite } \\
\text { remains }\end{array}$ & Calcite, Quartz & Trilobite-bearing limestone & XRD, EMPA & $\begin{array}{l}31^{\circ} 22^{\prime} 47.88^{\prime \prime} \mathrm{N} \\
4^{\circ} 3{ }^{\prime} 32.32^{\prime \prime W}\end{array}$ \\
\hline 23 & mound & Trilobite floatstone & Calcite, Quartz & Trilobite-bearing limestone & XRD, EMPA & $\begin{array}{l}31^{\circ} 22^{\prime} 44.58 " \mathrm{~N} \\
4^{\circ} 3^{\prime} 45.26^{\prime \prime} \mathrm{W}\end{array}$ \\
\hline 24 & mound & $\begin{array}{l}\text { Dark and grey micrite interbedded } \\
\text { with blocky and radiaxial calcite }\end{array}$ & Calcite, Quartz & Dark layered micrite & XRD & $\begin{array}{c}31^{\circ} 22^{\prime} 53.23^{\prime \prime} \mathrm{N} \\
4^{\circ} 1^{\prime} 49.44^{\prime \prime} \mathrm{W}\end{array}$ \\
\hline 25 & mound & $\begin{array}{l}\text { Isopachous and botryoidal fibrous } \\
\text { cements and blocky calcite }\end{array}$ & Calcite & Vein filling & $\begin{array}{l}\text { XRD, XRF, } \\
\text { ICP-MS }\end{array}$ & $\begin{array}{c}31^{\circ} 22^{\prime} 43.62^{\prime \prime} \mathrm{N} \\
4^{\circ} 2^{\prime} 24.66^{\prime \prime} \mathrm{W}\end{array}$ \\
\hline
\end{tabular}

*Based on XRD data integrated with EDX analyses.

and ultra polished disks (operating conditions: 15 and $25 \mathrm{kV}$ accelerating voltage). Ten ultra polished disks and three thin sections were investigated with an Electron Probe Micro Analyser (EPMA) at Universi ty of Johannesburg (see Supplementary materials S1, S2).

Sub samples for geochemical analyses were obtained by shattering polished slabs and collecting rock chips from the desired parts of the specimens. Small chips (ca. $100200 \mathrm{~g}$ ) of bulk specimens (avoiding weathered surfaces) were crushed in an agate mortar and then powdered using an agate mill. X ray diffraction (XRD) analyses (Table 1) were performed with a Philips PW 1130, CuK1, X ray diffractometer at the University of Bologna. Major and trace elements of thirteen samples (Table 2) were analyzed by $\mathrm{X}$ ray fluorescence using a PANanalytical Magix PRO on fusion disks at University of Johannesburg and by ICP MS on solutions using a Perkin Elmer Sciex ELANDRC e instrument at the University of the Witwatersrand (Table 2 ). For carbonates two distinct leaching procedures were performed (see Table 2): i) ca. $100 \mathrm{mg}$ of sample powder was leached with ultrapure hydrochloric acid in three consecutive steps using different normalities and volumes $(0.3 \mathrm{ml}$ of $0.4 \mathrm{~N}, 0.5 \mathrm{ml}$ of $2.5 \mathrm{~N}$ and $0.2 \mathrm{ml}$ of $6.1 \mathrm{~N} \mathrm{HCl})$ until no further reaction was noticed; ii) ca. $100 \mathrm{mg}$ of sample powder was leached with $1 \mathrm{ml}$ of ultrapure $1 \mathrm{~N}$ acetic acid. Leaching was undertaken at room temperature and while agitating the sample. Total reaction time was $2 \mathrm{~h}$ using $\mathrm{HCl}$ and $12 \mathrm{~h}$ using acetic acid. Insoluble residue from both preparations was separated by centrifuging. Samples 2 and 8 were leached with both hydrochloric acid and acetic acid. Results of both acidifications are reported for comparison in Table 2. Rongemaille et al. (2011) recommended the use of $5 \%(\mathrm{v} / \mathrm{v})$ acetic acid solution at room temperature for $24 \mathrm{~h}$ to achieve total leaching of the carbonate phases while avoiding dissolution of clastic material and Fe Mn oxides. Here we adopted both acetic acid and hydrochloric acid leaching. We used a similar acetic acid concentration (c. 6\%) as recommended by Rongemaille et al. (2011) and we evaluated potential contamination by comparing REE patterns with Fe and Mn contents and concentration of elements present in the clastic phase (cf. Nothdurft et al., 2004; Allwood et al., 2010; see Supplementary materials S3). Our results show that, for the material investigated, both leaching procedures 

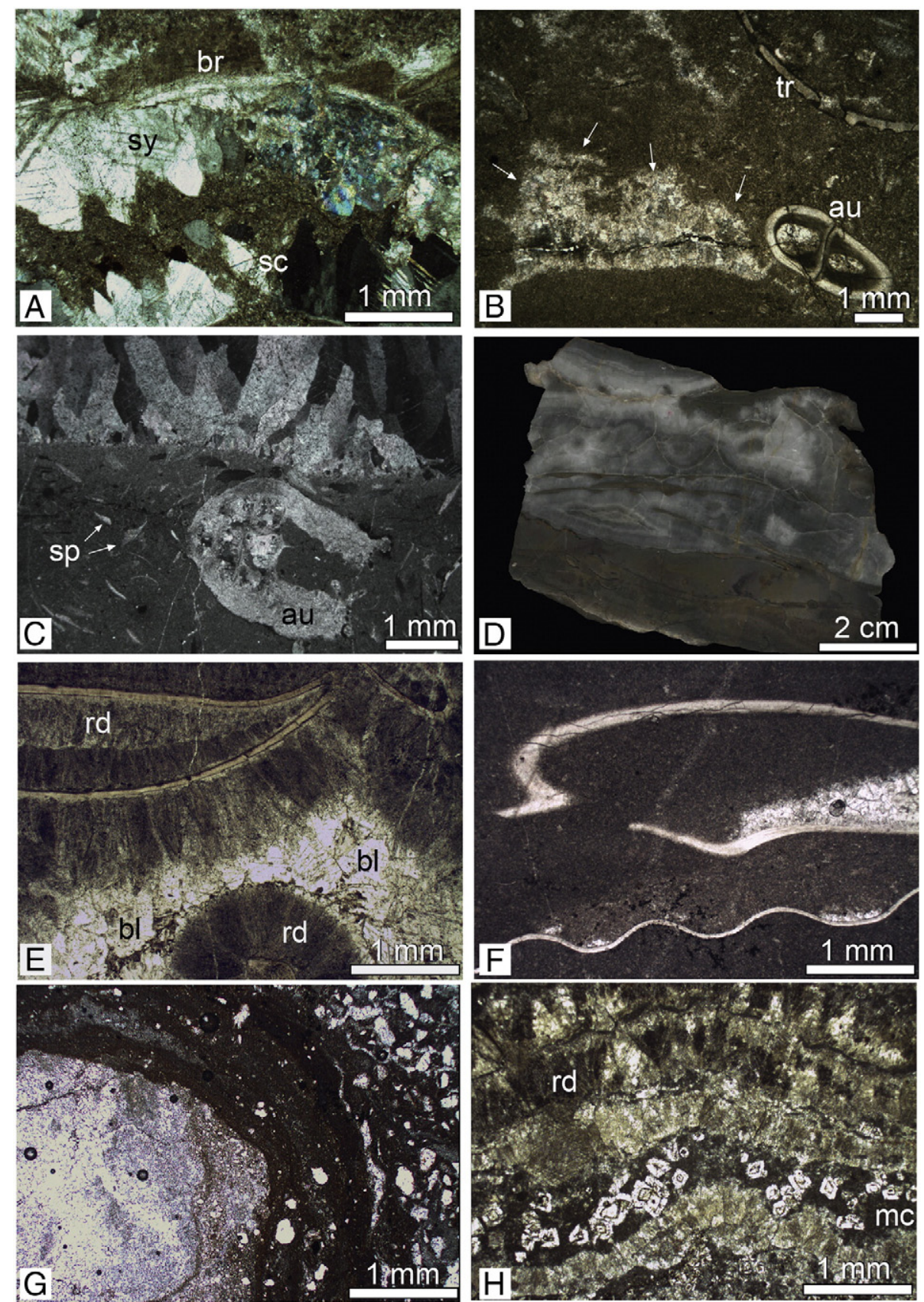

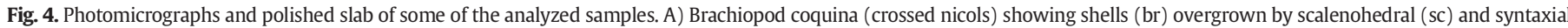

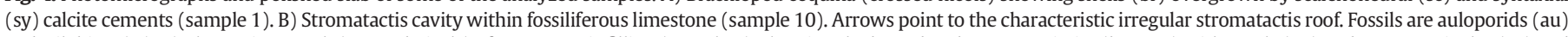

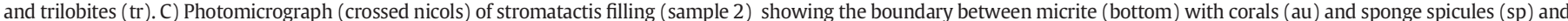

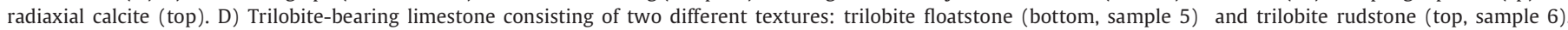

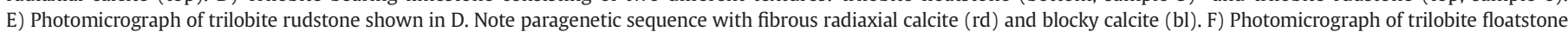

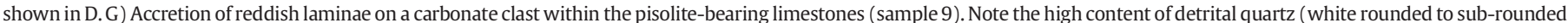
clasts) within the matrix. H) Paragenetic sequence of a fracture fill (sample 7) showing radiaxial fibrous (rd) calcite, micrite (mc), and dolomite rhombohedra (bright crystals).

adopted brought into solution mainly the carbonate phases resulting in only minor terrigenous and/or oxide contamination ( $\S 5.1$ ). For non carbonate samples 11 and 12 (Table 1 ), $50 \mathrm{mg}$ of powdered sample was dissolved in a mixture of $3: 2 \mathrm{HF}$ and $\mathrm{HNO}_{3}$ in a high pressure Teflon vessel in a CEM Mars Express Microwave digester with 40 min digestion times and evaporated to dryness in Teflon beakers before being taken up in 5\% $\mathrm{HNO}_{3}$ for analysis. Solutions were made up to $50 \mathrm{ml}$ for analysis. Internal standards (10 ppb Rh, In, Re and $\mathrm{Bi}$ ) and calibration solutions were prepared from certified single and multi element standard solutions. The instrument was tuned to avoid interference by oxides and doubly charged species so that $\mathrm{Ba}^{2+} / \mathrm{Ba}$ and $\mathrm{CeO} / \mathrm{Ce}$ were $<0.03$. Quality of data was monitored using the international standards BCR 1, BHVO 1, and BIR 1 .

We calculated LREE fractionation, ¿REE, Ce anomaly, Pr anomaly, Eu anomaly, Gd anomaly, Y/Ho ratio (Table 4), and the relation between these proxies and trace and transition elements. For ease of comparison with recent work on Paleozoic carbonate REE patterns we normalized REE data to post Archean average Australian shale (PAAS, Nance and Taylor, 1976; Taylor and McLennan, 1985) regarded to approximate the composition of average terrigenous input to the oceanic environment. 
Table 2

Major element contents (in wt. \%) and trace element concentrations (in mg/kg) for SRG samples. Samples leached with acetic acid are in gray.

\begin{tabular}{|c|c|c|c|c|c|c|c|c|c|c|c|c|c|c|c|}
\hline Sample & KK1 & KK2 & & KK5 & KK6 & KK7 & \multicolumn{2}{|c|}{ KK8 } & KK9 & KK10 & KK11 & KK12 & KK20 & KK21 & KK25 \\
\hline $\mathrm{Al}_{2} \mathrm{O}_{3}$ & 0.96 & \multicolumn{2}{|c|}{1.28} & 0.25 & 0.16 & 0.13 & \multicolumn{2}{|c|}{0.80} & 1.24 & 1.89 & 0.10 & 0.15 & 0.75 & 0.11 & 0.30 \\
\hline $\mathrm{CaO}$ & 54.16 & \multicolumn{2}{|c|}{53.10} & 55.51 & 55.45 & 54.84 & \multicolumn{2}{|c|}{53.44} & 45.90 & 52.17 & 0.57 & 0.25 & 52.70 & 55.77 & 55.64 \\
\hline $\mathrm{Fe}_{2} \mathrm{O}_{3}$ & 0.28 & \multicolumn{2}{|c|}{0.72} & 0.36 & 0.12 & 0.20 & \multicolumn{2}{|c|}{0.27} & 0.54 & 0.48 & 0.07 & 87.17 & 0.37 & 0.24 & 0.24 \\
\hline $\mathrm{K}_{2} \mathrm{O}$ & 0.18 & \multicolumn{2}{|c|}{0.25} & 0.06 & - & - & \multicolumn{2}{|c|}{0.18} & 0.22 & 0.47 & - & - & 0.18 & - & 0.07 \\
\hline $\mathrm{MgO}$ & 0.63 & \multicolumn{2}{|c|}{0.58} & 0.60 & 0.69 & 1.37 & \multicolumn{2}{|c|}{0.45} & 0.31 & 0.62 & - & 0.14 & 0.53 & 0.51 & 0.43 \\
\hline $\mathrm{MnO}$ & 0.05 & \multicolumn{2}{|c|}{0.07} & - & 0.06 & - & \multicolumn{2}{|c|}{0.06} & - & 0.06 & - & - & 0.20 & 0.08 & 0.16 \\
\hline $\mathrm{Na}_{2} \mathrm{O}$ & - & \multicolumn{2}{|c|}{0.13} & - & - & - & \multicolumn{2}{|c|}{ - } & - & - & - & - & - & - & - \\
\hline $\mathrm{SiO}_{2}$ & 1.52 & \multicolumn{2}{|c|}{2.51} & 1.95 & 0.28 & 0.20 & \multicolumn{2}{|c|}{1.22} & 14.73 & 2.98 & 97.67 & 1.17 & 3.64 & 0.46 & 0.41 \\
\hline $\mathrm{TiO}_{2}$ & 0.05 & \multicolumn{2}{|c|}{0.08} & - & - & - & & & 0.08 & 0.12 & - & - & - & - & - \\
\hline LOI & 40.64 & 41 & & 40.89 & 42.24 & 43.09 & & & 36.09 & 41.14 & 0.65 & 10.40 & 40.56 & 41.90 & 41.92 \\
\hline Total & 98.46 & 100 & & 99.68 & 99.08 & 99.89 & 102 & & 99.11 & 100.04 & 99.06 & 99.4 & 98.93 & 99.14 & 99.17 \\
\hline $\mathrm{Li}$ & 0.84 & 0.31 & 0.20 & 0.25 & 0.44 & 0.43 & 0.67 & 0.35 & 2.26 & 0.76 & 15.94 & 0.27 & 0.64 & 0.29 & 0.13 \\
\hline $\mathrm{P}$ & 38.7 & 115.7 & 19.7 & 138.7 & 275.7 & 262.8 & 110.0 & 9.5 & 143.7 & 38.6 & 42.3 & 482.3 & 226.9 & 417.0 & 29.0 \\
\hline Sc & 1.03 & 2.71 & 1.58 & 2.21 & 3.49 & 5.63 & 8.07 & 0.70 & 8.69 & 2.80 & 0.02 & 0.12 & 12.00 & 11.81 & 0.28 \\
\hline $\mathrm{Ti}$ & 0.56 & 4.33 & 0.91 & 0.79 & 0.98 & 1.07 & 1.63 & 0.45 & 4.05 & 0.48 & 14.79 & 38.27 & 1.48 & 1.17 & 0.39 \\
\hline V & 2.76 & 0.98 & 0.53 & 1.23 & 0.34 & 0.90 & 20.31 & 10.21 & 3.43 & 24.03 & 4.73 & 128.81 & 12.93 & 1.96 & 0.78 \\
\hline $\mathrm{Cr}$ & 0.93 & 1.06 & 0.86 & 1.27 & 1.66 & 1.45 & 4.42 & 2.53 & 3.05 & 1.85 & 1.16 & 8.32 & 3.40 & 2.60 & 0.42 \\
\hline Co & 3.38 & 2.85 & 3.68 & 2.59 & 2.51 & 3.27 & 12.49 & 10.74 & 4.61 & 4.38 & 0.17 & 12.79 & 38.63 & 7.39 & 2.71 \\
\hline $\mathrm{Ni}$ & 34.3 & 33.3 & 36.9 & 34.0 & 35.6 & 41.0 & 53.2 & 41.3 & 39.6 & 48.5 & 2.4 & 67.2 & 97.4 & 51.0 & 32.8 \\
\hline $\mathrm{Cu}$ & 0.31 & 0.95 & 0.26 & 0.41 & 0.47 & 0.92 & 81.61 & 37.31 & 1.05 & 0.25 & 0.72 & 3.60 & 31.67 & 4.51 & 0.20 \\
\hline $\mathrm{Zn}$ & 1.80 & 3.54 & 2.48 & 7.08 & 10.72 & 5.21 & 20.97 & 9.22 & 2.46 & 4.00 & 40.29 & 703.97 & 32.44 & 6.71 & 0.98 \\
\hline $\mathrm{Ga}$ & 0.05 & 0.15 & 0.08 & 0.11 & 0.12 & 0.06 & 0.22 & 0.05 & 0.21 & 0.18 & 1.86 & 3.53 & 0.39 & 0.21 & 0.07 \\
\hline As & 0.12 & 0.07 & 0.05 & 0.06 & 0.04 & 0.05 & 1.51 & 0.77 & 0.14 & 0.09 & 0.07 & 367.46 & 0.26 & 0.12 & 0.03 \\
\hline $\mathrm{Rb}$ & 0.13 & 0.23 & 0.13 & 0.11 & 0.09 & 0.09 & 0.27 & 0.16 & 0.37 & 0.37 & 0.37 & 0.10 & 0.28 & 0.10 & 0.02 \\
\hline $\mathrm{Sr}$ & 117.3 & 150.8 & 188.1 & 134.8 & 158.8 & 285.6 & 295.3 & 334.5 & 62.2 & 207.2 & 3.9 & 6.3 & 205.7 & 166.4 & 327.1 \\
\hline $\mathrm{Y}$ & 2.77 & 6.06 & 6.87 & 18.82 & 6.72 & 6.07 & 2.43 & 2.59 & 2.61 & 15.83 & 0.26 & 6.16 & 17.49 & 10.24 & 1.06 \\
\hline $\mathrm{Zr}$ & 0.07 & 0.55 & 0.22 & 0.82 & 0.80 & 0.46 & 0.59 & 0.08 & 0.55 & 0.29 & 0.66 & 5.76 & 1.26 & 0.45 & 0.15 \\
\hline $\mathrm{Ba}$ & 41.8 & 47.7 & 58.4 & 12.6 & 5.5 & 22.2 & 244.9 & 108.7 & 48.9 & 31.9 & 141.2 & 5.0 & 25.3 & 23.6 & 48.7 \\
\hline $\mathrm{La}$ & 1.96 & 2.23 & 2.96 & 4.25 & 1.37 & 1.11 & 0.55 & 0.73 & 1.31 & 8.09 & 0.27 & 1.57 & 2.63 & 1.30 & 0.54 \\
\hline $\mathrm{Ce}$ & 3.33 & 4.44 & 5.52 & 3.43 & 5.76 & 0.34 & 1.05 & 1.59 & 1.93 & 19.37 & 0.33 & 2.41 & 1.98 & 4.45 & 1.19 \\
\hline $\operatorname{Pr}$ & 0.429 & 0.667 & 0.829 & 0.786 & 0.302 & 0.178 & 0.150 & 0.234 & 0.388 & 1.953 & 0.049 & 0.400 & 0.633 & 0.261 & 0.151 \\
\hline $\mathrm{Nd}$ & 1.768 & 3.077 & 3.601 & 3.534 & 1.565 & 0.799 & 0.791 & 1.150 & 1.730 & 8.119 & 0.217 & 1.852 & 3.013 & 1.311 & 0.700 \\
\hline $\mathrm{Sm}$ & 0.399 & 0.688 & 0.842 & 0.788 & 0.377 & 0.180 & 0.347 & 0.387 & 0.429 & 1.944 & 0.131 & 0.447 & 0.825 & 0.330 & 0.201 \\
\hline $\mathrm{Eu}$ & 0.101 & 0.160 & 0.201 & 0.185 & 0.083 & 0.048 & 0.122 & 0.128 & 0.102 & 0.422 & 0.105 & 0.114 & 0.190 & 0.084 & 0.064 \\
\hline Gd & 0.411 & 0.754 & 0.863 & 1.134 & 0.574 & 0.264 & 0.262 & 0.305 & 0.456 & 2.298 & 0.046 & 0.668 & 1.074 & 0.583 & 0.183 \\
\hline $\mathrm{Tb}$ & 0.058 & 0.107 & 0.127 & 0.178 & 0.076 & 0.038 & 0.037 & 0.044 & 0.063 & 0.335 & 0.003 & 0.095 & 0.168 & 0.079 & 0.019 \\
\hline Dy & 0.370 & 0.668 & 0.786 & 1.292 & 0.494 & 0.279 & 0.233 & 0.284 & 0.344 & 1.999 & 0.022 & 0.637 & 1.171 & 0.562 & 0.130 \\
\hline Ho & 0.073 & 0.129 & 0.165 & 0.296 & 0.104 & 0.070 & 0.046 & 0.055 & 0.062 & 0.415 & 0.004 & 0.138 & 0.257 & 0.124 & 0.022 \\
\hline $\mathrm{Er}$ & 0.218 & 0.358 & 0.451 & 0.875 & 0.285 & 0.217 & 0.126 & 0.153 & 0.162 & 1.163 & 0.013 & 0.388 & 0.740 & 0.360 & 0.065 \\
\hline $\mathrm{Tm}$ & 0.031 & 0.050 & 0.062 & 0.123 & 0.038 & 0.029 & 0.017 & 0.022 & 0.020 & 0.170 & 0.002 & 0.056 & 0.099 & 0.049 & 0.008 \\
\hline $\mathrm{Yb}$ & 0.214 & 0.319 & 0.396 & 0.797 & 0.254 & 0.197 & 0.109 & 0.140 & 0.123 & 1.061 & 0.010 & 0.358 & 0.683 & 0.324 & 0.043 \\
\hline $\mathrm{Lu}$ & 0.029 & 0.045 & 0.055 & 0.116 & 0.036 & 0.030 & 0.015 & 0.017 & 0.016 & 0.158 & 0.002 & 0.052 & 0.096 & 0.046 & 0.004 \\
\hline $\mathrm{Pb}$ & 2.15 & 2.35 & 2.24 & 1.80 & 1.32 & 0.75 & 13.62 & 10.91 & 1.32 & 3.69 & 3.52 & 202.33 & 6.53 & 25.59 & 2.02 \\
\hline Th & 0.31 & 0.64 & 0.51 & 0.19 & 0.12 & 0.05 & 0.20 & 0.18 & 0.11 & 1.87 & 0.04 & 0.28 & 0.35 & 0.04 & 0.04 \\
\hline U & 6.57 & 0.43 & 0.43 & 0.97 & 0.27 & 0.08 & 1.85 & 2.32 & 0.23 & 18.79 & 2.03 & 1.25 & 0.85 & 0.14 & 0.62 \\
\hline$\sum \mathrm{REE}$ & 9.39 & 13.69 & 16.86 & 17.78 & 11.32 & 3.78 & 3.86 & 5.24 & 7.13 & 47.50 & 1.20 & 9.18 & 13.56 & 9.86 & 3.32 \\
\hline
\end{tabular}

Fractionation of LREE was calculated as $\mathrm{Pr}_{\mathrm{SN}} / \mathrm{Yb}_{\mathrm{SN}}$ to avoid anoma lous La and Ce concentrations encountered in seawater. Normalized $\mathrm{La}, \mathrm{Ce}, \mathrm{Eu}$, and Gd anomalies were calculated following the methods of Bau and Dulski (1996), Webb and Kamber (2000); Bolhar et al. (2004):
$\mathrm{La} / \mathrm{La}^{*} \quad[\mathrm{La} /(3 \mathrm{Pr} 2 \mathrm{Nd})]_{\mathrm{SN}}$
$\mathrm{Ce} / \mathrm{Ce}^{*} \quad[\mathrm{Ce} /(0.5 \mathrm{La}+0.5 \mathrm{Pr})]_{\mathrm{SN}}$

$\mathrm{Eu} / \mathrm{Eu}^{*} \quad[\mathrm{Eu} /(0.67 \mathrm{Sm}+0.33 \mathrm{~Tb})]_{\mathrm{SN}}$

$\mathrm{Gd} / \mathrm{Gd}^{*} \quad[\mathrm{Gd} /(0.33 \mathrm{Sm}+0.67 \mathrm{~Tb})]_{\mathrm{SN}}$.

The occurrence of positive La anomalies in seawater (e.g. de Baar et al., 1991) and marine precipitates may result in calculation of an incorrect Ce anomaly where Ce* is calculated following Eq. (2). To 
Table 3

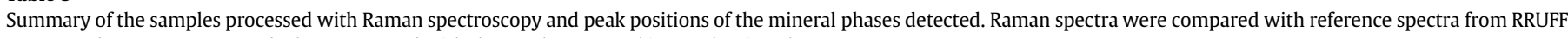
Da-taBase by Downs, 2006 andWhite, 2009 and with the results reported in Cavalazzi et al., 2012.

\begin{tabular}{|c|c|c|c|c|}
\hline Sample & Provenance & Facies description & Mineral phase & Peaks \\
\hline \multirow[t]{3}{*}{5} & \multirow[t]{3}{*}{ Mound } & \multirow[t]{3}{*}{ Trilobite floatstone } & Calcite & $156,281,711,1086 \mathrm{~cm}^{1}$ \\
\hline & & & Quartz & $128,208,466 \mathrm{~cm}^{1}$ \\
\hline & & & Carbonaceous matter & $\mathrm{D} 1: 1350 \mathrm{~cm}^{1} ; \mathrm{G} 2: 1600 \mathrm{~cm}^{1}$ \\
\hline \multirow[t]{4}{*}{6} & \multirow[t]{4}{*}{ Mound } & \multirow[t]{4}{*}{ Trilobite rudstone } & Calcite & $156,281,711,1086 \mathrm{~cm}^{1}$ \\
\hline & & & Quartz & $128,208,466 \mathrm{~cm}^{1}$ \\
\hline & & & Carbonaceous matter & $\mathrm{D} 1: 1350 \mathrm{~cm}^{1} ; \mathrm{G} 2: 1600 \mathrm{~cm}^{1}$ \\
\hline & & & Goethite & $244,300,398,480,553,684,1002,1118,1303 \mathrm{~cm}^{1}$ \\
\hline \multirow[t]{2}{*}{8} & \multirow[t]{2}{*}{ Mound } & \multirow[t]{2}{*}{ Dark layered micrite } & Calcite & $156,281,711,1086 \mathrm{~cm}^{1}$ \\
\hline & & & Carbonaceous matter & $\mathrm{D} 1: 1350 \mathrm{~cm}^{1} ; \mathrm{G} 2: 1600 \mathrm{~cm}^{1}$ \\
\hline 11 & Vent & Quartz-bearing & Quartz & $128,208,466 \mathrm{~cm}^{1}$ \\
\hline 12 & Vent & Goethite & Goethite & $244,300,398,480,553,684,1002,1118,1303 \mathrm{~cm}^{1}$ \\
\hline
\end{tabular}

avoid this problem Bau and Dulski (1996) accounted for the possibility of positive La anomalies by comparing Eq. (2) to

$\left(\operatorname{Pr} / \operatorname{Pr}^{*}\right)_{S N}[\operatorname{Pr} /(0.5 \mathrm{Ce}+0.5 \mathrm{Nd})]_{S N}$

(see also Webb and Kamber, 2000). Where $\left(\operatorname{Pr} / \operatorname{Pr}^{*}\right)_{S N}>1$, a negative Ce anomaly exists; where $\left(\mathrm{Ce} / \mathrm{Ce}^{*}\right)_{\mathrm{SN}}<1$, a positive La anomaly exists. Ce anomalies were then calculated for comparison with the method given by Bolhar et al. (2004):

$\mathrm{Ce} / \mathrm{Ce}^{*} \quad[\mathrm{Ce} /(2 \mathrm{Pr} \mathrm{Nd})]_{\mathrm{SN}}$.

Although this latter method reflects anomalously high Ce contents, $\mathrm{Ce} / \mathrm{Ce}^{*}$ values are less affected by La anomalies and thus will be com pared with those obtained by other methods.

The REE abundances in Early Devonian seawater were estimated from the partition coefficients given by Tanaka and Kawabe (2006):

$\mathrm{Kd}(\mathrm{REE})_{\text {seawater }} \quad([\mathrm{REE}] /[\mathrm{Ca}])_{\text {limestone }} /([\mathrm{REE}] /[\mathrm{Ca}])_{\text {seawater }}$.

Following this method we estimated the REE abundances in Devoni an seawater by considering an average of REE data from SRG limestones and the absolute values of partition coefficients (Kd) under water conditions of $\mathrm{pH}=8.2,\left[\mathrm{CO}_{2}\right]_{\mathrm{TOT}}=0.00235$, salinity $=$ 35\% (Table 4 in Tanaka and Kawabe, 2006).

\section{Results}

\subsection{Mineralogy and major element composition}

\subsubsection{Mound facies}

Samples from the mound facies (Fig. 1) consist of low Mg calcite with subordinate dolomite, quartz, and goethite (Table 1). Particularly, within stromatactis and trilobite bearing limestones (samples 2, 5, 22 24) XRD analyses revealed minor dolomite and quartz contents. Be sides calcite and disordered carbonaceous matter, Raman spectroscopy revealed quartz and goethite (Table 3) associated with micrite filling trilobite shells within rudstone (Fig. 5A B). Minor amounts of carbo naceous matter were also detected within dark layered limestones (sample 8; Fig. 5C).

Within limestones from the mound facies $\mathrm{Al}_{2} \mathrm{O}_{3}$ contents range be tween 0.11 and $1.89 \%$ (average $0.72 \%$ ); the highest value is from fossil iferous limestones (sample 10). The $\mathrm{MgO}$ contents within mound facies samples range between 0.43 and $0.69 \%$ (Table 2), while $\mathrm{SiO}_{2}$ contents have a range of $0.282 .98 \%$ showing the lowest values within vein fills (samples 21 and 25) and trilobite rudstone (sample 6). Within lime stones from the mound facies $\mathrm{K}_{2} \mathrm{O}$ and $\mathrm{MnO}$ contents are very low and other oxide contents are mostly below the XRF detection limit. Element concentrations of each sample (see EPMA Supplementary material S1) reveal rare peaks of $\mathrm{MgO}$ and $\mathrm{SiO}_{2}$ and a slight enrichment of $\mathrm{SiO}_{2}$ around trilobite cuticles. Trilobite bearing limestones show variation of chemical composition, both in floatstone and rudstone, with scattered $\mathrm{CaO}, \mathrm{FeO}$, and $\mathrm{SiO}_{2}$ peaks (see Supplementary material S2). Back scattered electron images of trilobite rudstone (Fig. 6A) revealed that the low $\mathrm{Mg}$ calcite of the cuticles was replaced by neomorphic patchy quartz.

Table 4

LREE depletion, Y/Ho ratios, shale-normalized anomalies, total REE contents, and stable isotope (C and O) compositions of studied samples.

\begin{tabular}{|c|c|c|c|c|c|c|c|c|c|c|}
\hline \multirow[t]{2}{*}{ SAMPLE } & \multirow{2}{*}{$\begin{array}{l}\text { LREE depletion } \\
\left(\operatorname{Pr}_{\mathrm{SN}} / \mathrm{Yb}_{\mathrm{SN}}\right)\end{array}$} & \multirow{2}{*}{$\begin{array}{l}\mathrm{Y} / \mathrm{Ho} \\
\text { ratio }\end{array}$} & \multicolumn{2}{|l|}{$\left(\mathrm{Ce} / \mathrm{Ce}^{*}\right)$} & \multirow{2}{*}{$\left(\mathrm{Pr} / \mathrm{Pr}{ }^{*}\right)$} & \multirow{2}{*}{$\frac{\left(\mathrm{Eu} / \mathrm{Eu}^{*}\right)}{[\mathrm{Eu} /(0.67 \mathrm{Sm}+0.33 \mathrm{~Tb})]_{\mathrm{SN}}}$} & \multirow[t]{2}{*}{$\left(\mathrm{Gd} / \mathrm{Gd}^{*}\right)$} & \multirow[t]{2}{*}{$\Sigma R^{\prime} E^{b}$} & \multirow{2}{*}{$\begin{array}{l}\delta^{13} \mathrm{C} \\
(\% \circ)^{\mathrm{c}}\end{array}$} & \multirow{2}{*}{$\begin{array}{l}\delta^{18} \mathrm{O} \\
(\% \circ)^{\mathrm{C}}\end{array}$} \\
\hline & & & {$[\mathrm{Ce} /(2 \mathrm{Pr}-\mathrm{Nd})]_{\mathrm{SN}}$} & {$\left[\mathrm{Ce}_{\mathrm{SN}} /\left(\mathrm{La}_{\mathrm{SN}}+\mathrm{Pr}_{\mathrm{SN}}\right) 1 / 2\right]$} & & & & & & \\
\hline $1^{\mathrm{a}}$ & 0.630 & 38.240 & 1.013 & 0.834 & 0.995 & 1.272 & 1.330 & 9.391 & 1.17 & 5.17 \\
\hline 2 & 0.658 & 46.969 & 1.032 & 0.829 & 0.989 & 1.135 & 1.343 & 13.690 & 0.43 & 10.00 \\
\hline 5 & 0.310 & 63.595 & 0.647 & 0.428 & 1.152 & 0.986 & 1.341 & 17.778 & 0.19 & 5.85 \\
\hline 6 & 0.374 & 64.654 & 3.796 & 2.055 & 0.561 & 0.971 & 1.547 & 11.317 & 0.85 & 8.61 \\
\hline 7 & 0.284 & 86.657 & 0.281 & 0.172 & 1.370 & 1.154 & 1.439 & 3.778 & N.D. & N.D. \\
\hline $8^{\mathrm{a}}$ & 0.525 & 47.085 & 1.200 & 0.875 & 0.941 & 1.790 & 1.191 & 5.235 & 10.08 & 10.13 \\
\hline 9 & 0.992 & 42.032 & 0.727 & 0.617 & 1.116 & 1.184 & 1.355 & 7.134 & 6.13 & 9.07 \\
\hline $10^{\mathrm{a}}$ & 0.579 & 38.146 & 1.308 & 1.120 & 0.885 & 1.021 & 1.349 & 47.502 & 0.53 & 10.38 \\
\hline 11 & 1.542 & 65.250 & 0.966 & 0.647 & 1.013 & 5.629 & 1.060 & 1.202 & N.D. & N.D. \\
\hline 12 & 0.352 & 44.645 & 0.942 & 0.700 & 1.021 & 1.100 & 1.459 & 9.184 & N.D. & N.D. \\
\hline 20 & 0.292 & 68.062 & 0.515 & 0.353 & 1.196 & 1.012 & 1.313 & 13.561 & 2.10 & 6.05 \\
\hline 21 & 0.253 & 82.581 & 3.145 & 1.749 & 0.607 & 1.041 & 1.574 & 9.864 & 0.21 & 6.31 \\
\hline $25^{\mathrm{a}}$ & 1.108 & 48.738 & 1.221 & 0.951 & 0.927 & 1.792 & 1.520 & 3.316 & 12.23 & 5.48 \\
\hline
\end{tabular}

a Samples leached with acetic acid.

b Total REE contents $(\mathrm{mg} / \mathrm{kg})$ excluded

Y. ${ }^{c}$ Data from Franchi et al. (2014). 

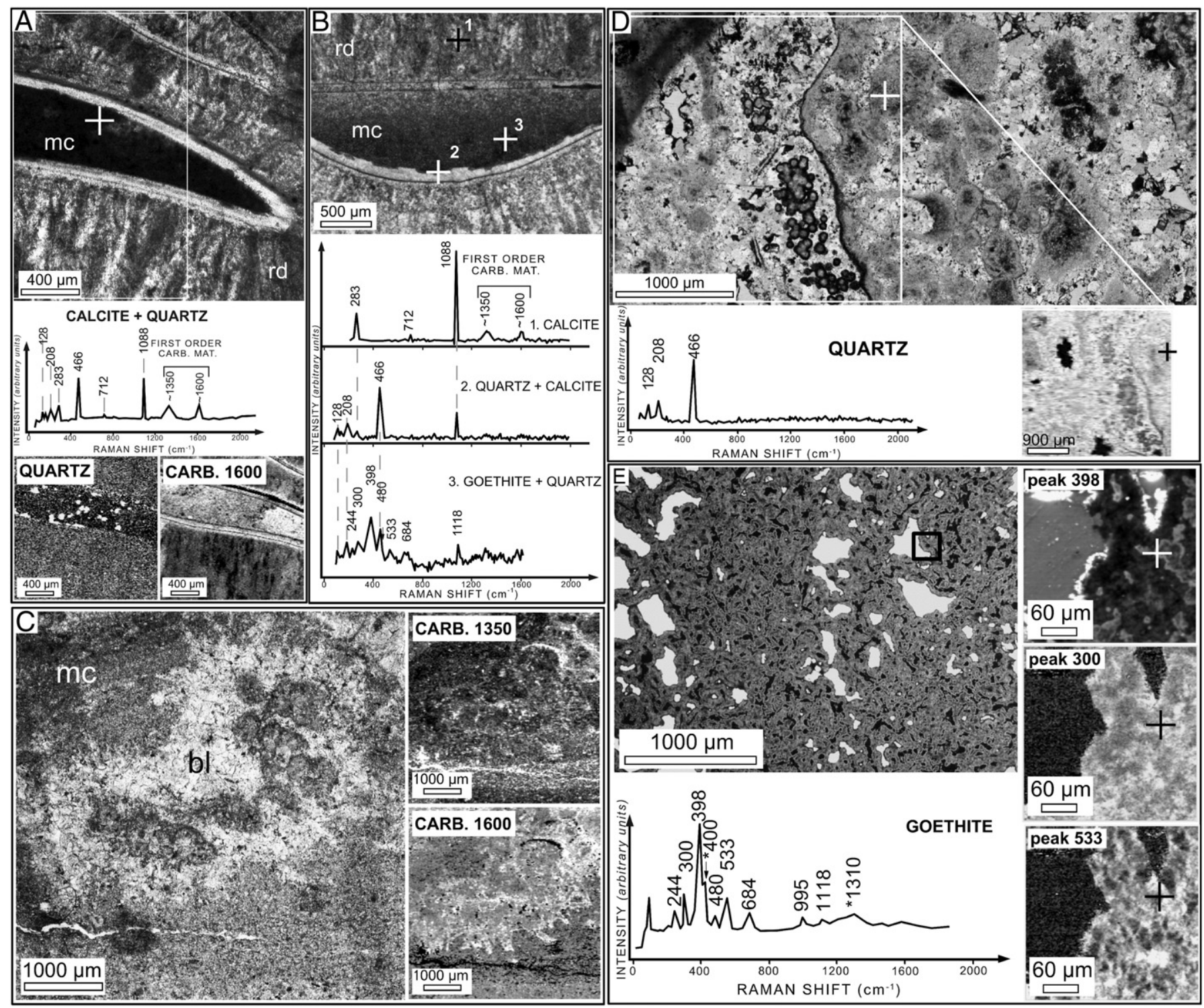

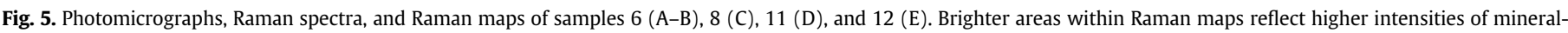

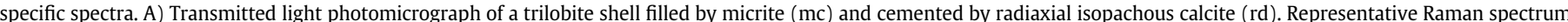

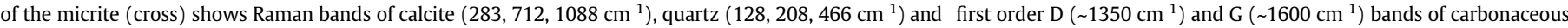

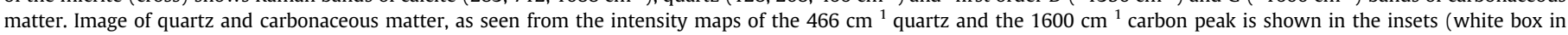

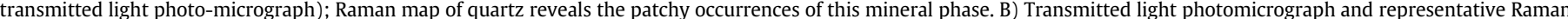

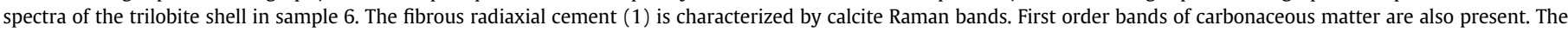

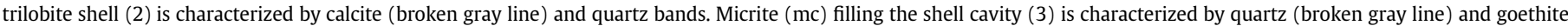

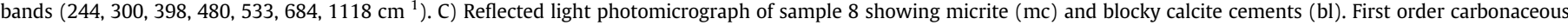

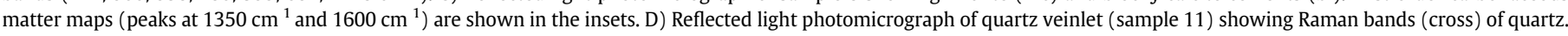

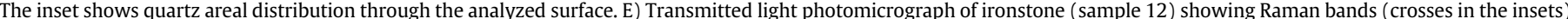

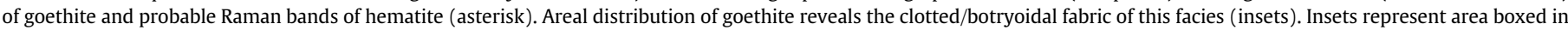
the transmitted light photomicrograph.

\subsubsection{Middle Seheb el Rhassel Group and inter mound facies}

Brachiopod coquina from the middle part of the SRG(Fig. 1) is dom inated by articulated shells cemented by syntaxial and scalenohedral calcite (Fig. 4A) with minor amounts of clastic quartz. Brachiopod coquina shows $\mathrm{Al}_{2} \mathrm{O}_{3}$ content below $1 \%$ and $\mathrm{SiO}_{2}$ of $1.5 \%$, whereas $\mathrm{MgO}$ content is about $0.7 \%$ and other oxides are below $0.5 \%$.

Relatively high quartz contents were detected within the intermound reddish pisolite limestone facies (samples 9, 15, 16, 19) and within tentaculite packstone (sample 20). Dykes are filled by calcite cement with minor amounts of dolomite (Fig. 4H).

Limestones from inter mound facies (Table 2) show $\mathrm{Al}_{2} \mathrm{O}_{3}$ contents of $0.131 .24 \%$, even though $\mathrm{Al}_{2} \mathrm{O}_{3}$ content changes consistently with facies. The highest $\mathrm{Al}_{2} \mathrm{O}_{3}$ content is from reddish pisolite bearing limestone. The $\mathrm{MgO}$ contents have a range of $0.141 .37 \%$; the highest value is from fractures fills that show evidence for inci$\mathrm{p}$ ient dolomitization (Fig. 4H). Fig. 6B shows back scattered electron micrograph of hypidiotopic dolomite cement within the fracture fills (same as Fig. $4 \mathrm{H}$ ). Due to the presence of detrital quartz the red dish pisolite bearing limestone is more enriched in $\mathrm{SiO}_{2}$ (14.73\%) compared to other samples from mound and intermound area which have $\mathrm{SiO}_{2}$ contents ranging between $0.20 \%$ and $3.64 \%$ (Tables 1,2 ). Lowest $\mathrm{SiO}_{2}$ contents have been detected within vein fills. Generally, $\mathrm{Fe}_{2} \mathrm{O}_{3}$ values are very low $(<1 \%)$ whereas other oxide contents are below the XRF detection limit. Results of EPMA line scans through samples from inter mound facies are shown in the Supplementary materials $(\mathrm{S} 1, \mathrm{~S} 2)$. 

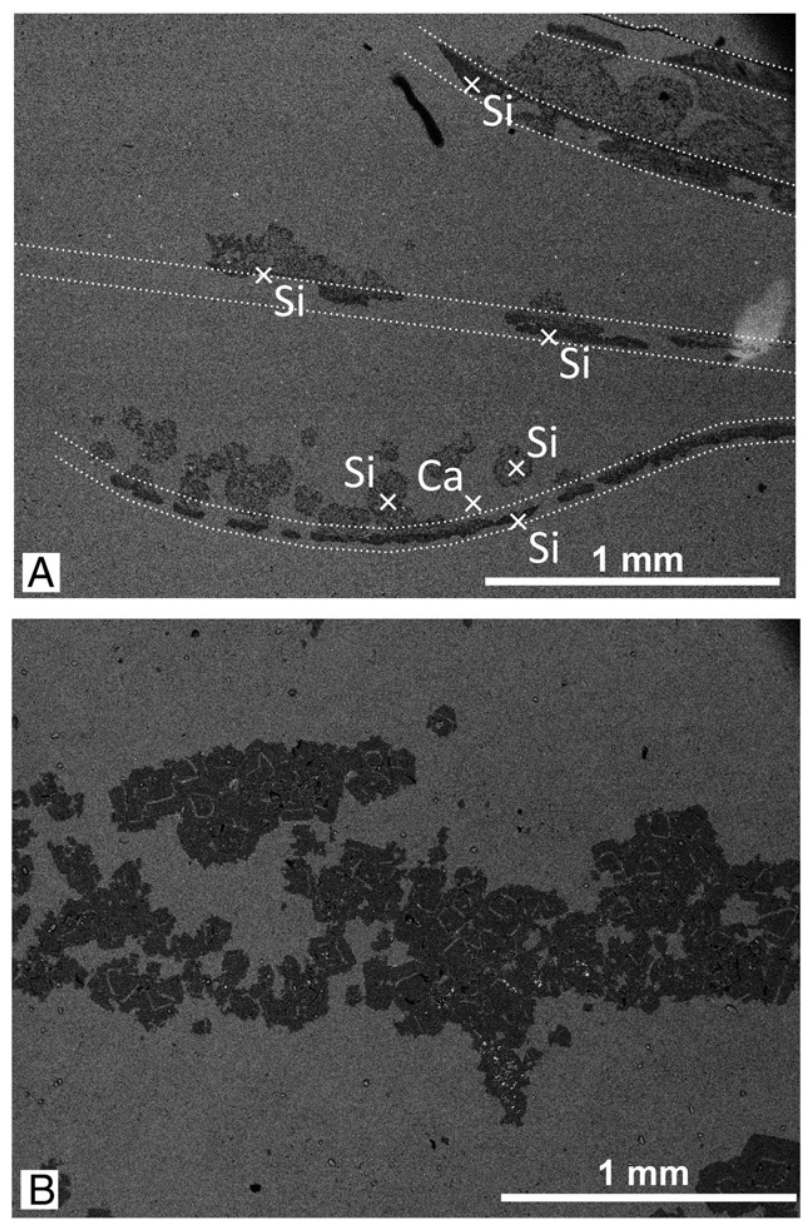

Fig. 6. Back scattered electron (BSE) micrographs of samples 5 (A) and 7 (B) and EPMA results (white crosses). A) Trilobite limestone showing patchy inclusion of quartz ( $\mathrm{Si}$ ) within trilobite cuticles (with dotted lines) and within trilobite fill. $\mathrm{Ca}=$ carbonates. $\mathrm{B}$ ) Vein filling carbonates showing a layer of Mg-rich (darker area) mineral phase (subhedral dolomite).

\subsubsection{Vent systems}

Ironstone collected from the upper part of the SRG consists of goethite $[\alpha \mathrm{FeO}(\mathrm{OH})]$ embedded in dolomitized limestone. Microcrys talline quartz veinlets occur within the goethite host rocks. The XRD analyses revealed that this silica phase is mainly $\alpha$ quartz + moganite $\left(\mathrm{SiO}_{2}\right)$. Microcrystalline quartz shows a peloidal fabric (Fig. 5D, insets). Representative Raman spectra of specimens from ironstone and veinlet fills (samples 11 and 12) contain distinctive goethite and quartz peaks (Table 3, Fig. 5D E), respectively. Raman maps of goethite highlighted the peculiar peloidal like fabric of this mineral (Fig. 5E).

Within the ironstone $\mathrm{Fe}_{2} \mathrm{O}_{3}$ content is $87.17 \%$ (Table 2), while $\mathrm{SiO}_{2}$ and $\mathrm{CaO}$ contents are $1.17 \%$ and $0.25 \%$, respectively. Although $\mathrm{As}, \mathrm{Cr}$, $\mathrm{Cu}, \mathrm{K}, \mathrm{Ni}$,and $\mathrm{Zn}$ were detected with EDS analyses (Fig. 7), other oxide contents are mostly below $0.2 \%$. Sample from veinlets shows a $\mathrm{SiO}_{2}$ content of $97.67 \%$, and minor amounts of $\mathrm{Al}_{2} \mathrm{O}_{3}$ and $\mathrm{Fe}_{2} \mathrm{O}_{3} ; \mathrm{CaO}$ content is $0.57 \%$ whereas other elements are below the detection limit.

\subsection{Trace elements and REE geochemistry}

Trace element concentrations are reported in Table 2 and PAAS normalized REE patterns are plotted in Figs. 8 and 9. Although general trends might be compared with shale normalized seawater REE pattern, the REE patterns within SRG limestones vary according to facies (Table 4; Fig. 8).
Total REE contents in the Seheb el Rhassel limestones range between $3.3 \mathrm{mg} / \mathrm{kg}$ and $47.5 \mathrm{mg} / \mathrm{kg}(9.5 \mathrm{mg} / \mathrm{kg}$ on average). The highest $\Sigma$ REE content was detected in the mound facies within fossiliferous limestone (sample 10). This sample also has the highest $U$ content of the data set ( $18.8 \mathrm{mg} / \mathrm{kg}$; see Discussion below). Floatstone and rudstone of the trilobite bearing facies have $\Sigma$ REE contents of 17.8 $\mathrm{mg} / \mathrm{kg}$ and $11.3 \mathrm{mg} / \mathrm{kg}$, respectively. Total REE contents within vein fills range between $3.3 \mathrm{mg} / \mathrm{kg}$ and $9.9 \mathrm{mg} / \mathrm{kg}$. Within limestones from mound and intermound facies the shale normalizedREE pattern typically shows LREE depletion compared to HREE $\left(\mathrm{Pr}_{\mathrm{SN}} / \mathrm{Yb}_{\mathrm{SN}}=0.30 .7\right)$. In particular, sample 9 shows HREE depletion compared to MREE $\left(\mathrm{Gd}_{\mathrm{SN}} / \mathrm{Yb}_{\mathrm{SN}}\right.$ $=2.5$ and $\mathrm{Dy}_{\mathrm{SN}} / \mathrm{Yb}_{\mathrm{SN}}=1.9$ ). Samples from both mound and intermoundfacies have a wide range of $\mathrm{Y} / \mathrm{Ho}$ ratios varying between 38.2 and 86.7; $\mathrm{Gd}$ anomalies are al ways positive (ranging between 1.2 and 1.6).

Fig. 10 reports Ce anomalies (calculated with the method proposed by Bau and Dulski, 1996) compared with Pr anomaly. Three groups of samples with distinct Ce anomaly (calculated following equation given by Bolhar et al., 2004) can be distinguished within the SRG limestones. The first group, which includes both samples from mound (sample 5) and intermound facies (samples 7, 9 and 20), shows a nega tive $\mathrm{Ce}$ anomaly (average $\mathrm{Ce} / \mathrm{Ce}^{*}=0.5$ ) coupled with slightly positive La anomaly. The second group, which includes mostly samples from mound facies (samples 2, 8, 10 and 25) and brachiopod coquina, lacks a real $\mathrm{Ce}$ anomaly (average $\mathrm{Ce} / \mathrm{Ce}^{*}=1.19$ ). The third group, which includes samples from the plumbing system from the inner part of the mounds ( 6 and 21 ), shows a pronounced positive $\mathrm{Ce}$ anomaly ( $\mathrm{Ce} / \mathrm{Ce}^{*}$ up to 3.8) coupled with a positive Gd anomaly ( $\mathrm{Gd} /$ $\mathrm{Gd}^{*} \sim 1.6$ ). Only samples from the second group (samples $1,2,8,10$, 25) show a positive $\mathrm{Eu}$ anomaly (average $\mathrm{Eu} / \mathrm{Eu}^{*}=1.2$ ). Particularly samples 8 and 25 have a strong positive Eu anomaly (up to 1.8). Eu anomalies within limestones from the upper part of the SRG correlate positively with $\mathrm{Ba}$ and $\mathrm{Sr}\left(\mathrm{R}^{2}=0.65\right.$ and $\mathrm{R}^{2}=0.45$, respectively; Fig. $11 \mathrm{~A}$ B). Considering both mound and intermound limestones (excluding outliers such as samples 8 and 25) Eu anomalies show a weak negative correlation with Ca (Fig. 11C) contents $\left(R^{2}=0.41\right)$. Whereas Ce anomalies show high negative correlation $\left(\mathrm{R}^{2}=0.78\right)$ with $\mathrm{P}_{2} \mathrm{O}_{5}$ contents (Fig. 11D).

Sample of vein quartz from the upper part of the SRG has the lowest $\Sigma$ REE content of the whole dataset $(1.2 \mathrm{mg} / \mathrm{kg})$, whereas goethite has $\Sigma$ REE content of $9.2 \mathrm{mg} / \mathrm{kg}$. Both samples lack a Ce anomaly. Vein quartz has the highest Eu anomaly in the dataset coupled with elevated Ba con tent (see Discussion). Goethite has the highest As content $(367 \mathrm{mg} / \mathrm{kg}$ ) which is 3 orders of magnitude higher than the limestone average $(0.2 \mathrm{mg} / \mathrm{kg})$. Ironstone is also enriched in $\mathrm{P}, \mathrm{V}, \mathrm{Zn}$, and $\mathrm{Pb}$ (Table 2).

\section{Discussion}

\subsection{Modification of the seawater REE signal}

Webb and Kamber (2000) showed that non skeletal carbonates incorporate REE in equilibrium with seawater. Further study demon strated that seawater REE patterns have hardly changed throughout the Phanerozoic (Shields and Webb, 2004) and HREE enrichment has been a characteristic feature of seawater since the late Archaean (Bau and Dulski, 1996; Kamber and Webb, 2001; Shields and Webb, 2004). In addition, REE in carbonate tend to be relatively immobile during diagenesis (Banner et al., 1988; Webb and Kamber, 2000; Nothdurft et al., 2004).

Mixing of seawater with other fluids, such as hydrothermal fluids of unknown composition, may have affected REE concentrations within SRG carbonates. For instance the carbonates could have formed over a range of physico chemical conditions that affected the REE distribu tions. Skeletal remains in both mound and inter mound facies were considered as parautochthonous bioclasts (Brachert et al., 1992 ) cemented by micrite, the precipitation of which was triggered by warm fluids mixing (Bełka, 1998; Mounji et al., 1998; Aitken et al., 


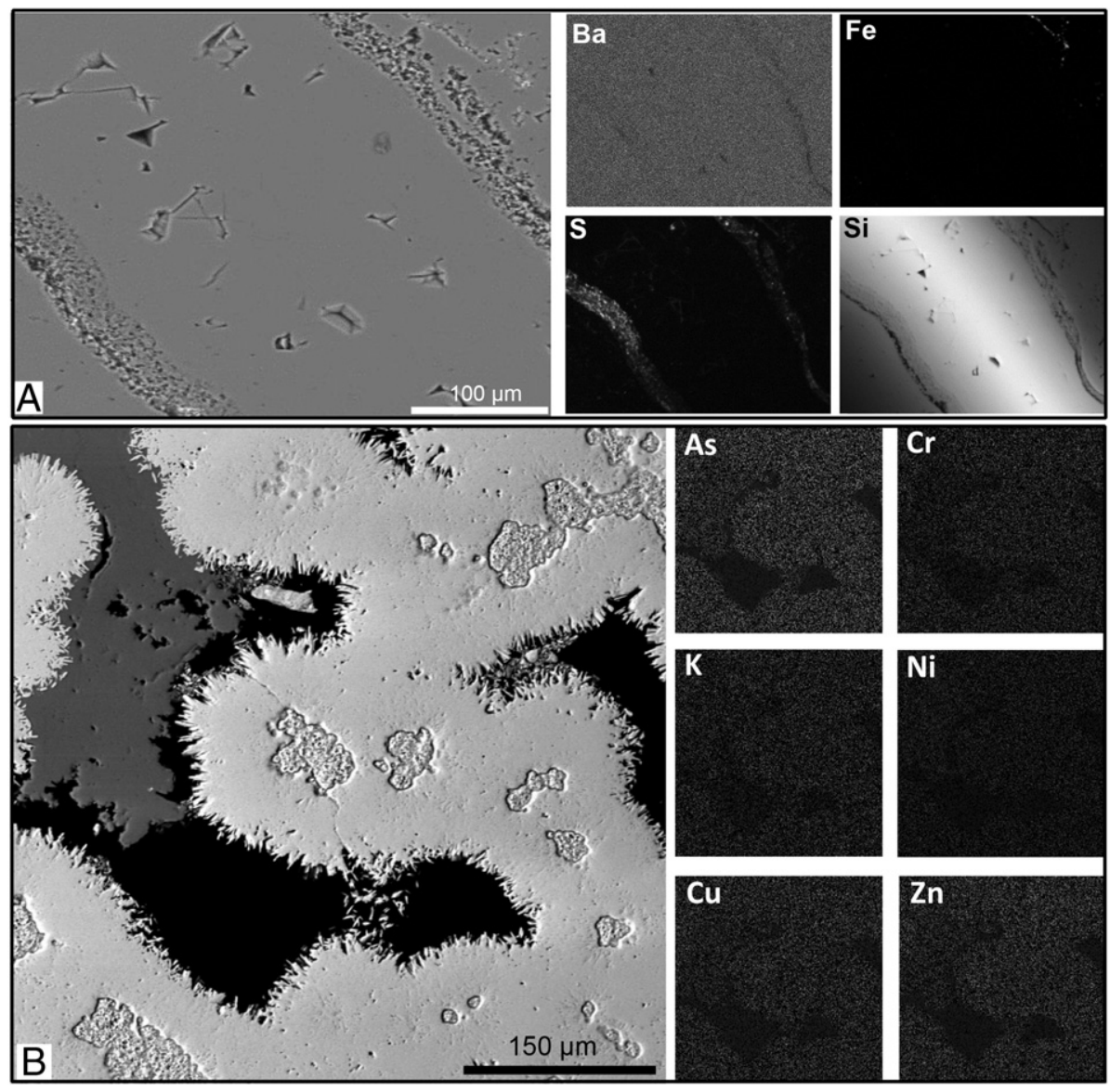

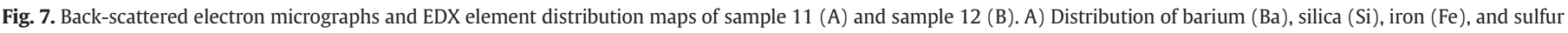

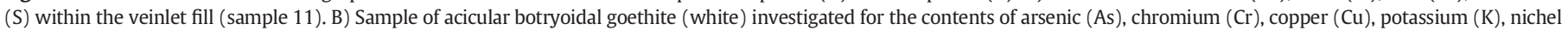
$(\mathrm{Ni})$, and zinc $(\mathrm{Zi})$. Dark and gray regions are vugs filled by epoxy resin.

2002; Franchi et al., 2014), followed by early lithification in shallow burial marine environment by pristine radiaxial fibrous calcite. The Kess Kess mounds were also affected by shallow burial diagenesis as suggested by the conspicuous presence of burial cements. Although we may assume that REE contents are constant within the carbonate lat tice, it needs to be considered that during early diagenesis variation of mineralogy has likely occurred. The fibrous radiaxial calcite could be a pseudomorph of pristine aragonite (Aitken et al., 2002) and therefore we need to assume that within the pore fluids geochemical exchanges occurred between different paragenetic phases. For this reason we per formed analyses on bulk samples to obtain average REE concentrations

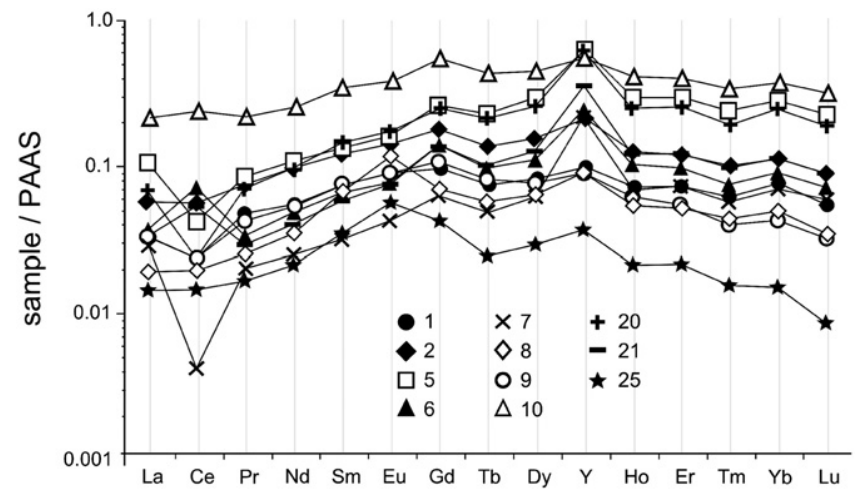

Fig. 8. PAAS-normalized (PAAS after Nance and Taylor, 1976) REE + Y plots of SRG limestones. for each sedimentological facies. Moreover, the facies related variations of REE fractionation might be considered as post hoc evidence of the consistency of the sampling methods applied in this work.

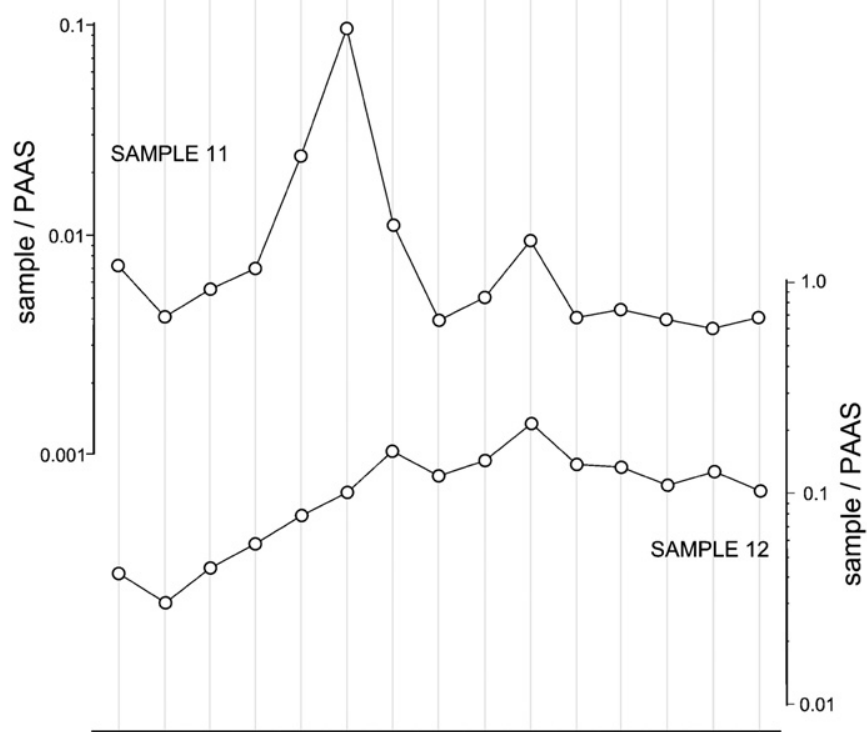

Fig. 9. PAAS-normalized REE + Y plots of ironstone (sample 12) and vein quartz (sample 11) of the SRG 


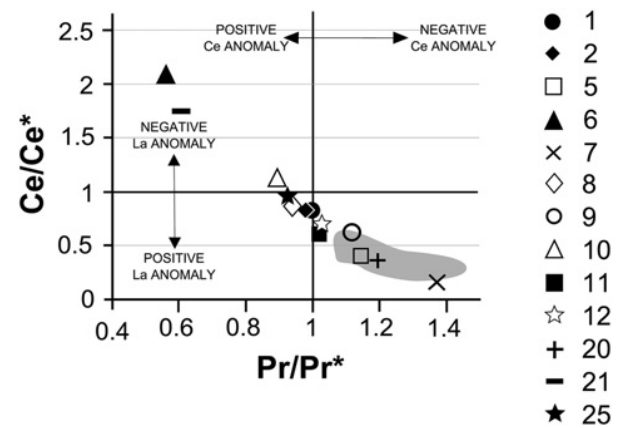

Fig. 10. Plot of PAAS-normalized Pr anomaly versus $\mathrm{Ce}$ anomaly ( $\mathrm{Ce} / \mathrm{Ce}^{*}=[\mathrm{Ce} /$ $\left.(0.5 \mathrm{La}+0.5 \mathrm{Pr})]_{\mathrm{SN}}\right)$ using the graphic method of Bau and Dulski (1996) (modified from Webb and Kamber, 2000). This plot discriminates between La and Ce anomalies, which both may be present in seawater. The range of modern seawater is represented by the shaded area (after Nothdurft et al., 2004). Note that only samples 5, 7, 9, and 20 plot within the range of modern seawater. carbonates as a proxy for the REE content of ancient seawater is compli avoided terrigenous contamination of the carbonate phase REE patterns. cated by possible modification due to the presence of 1) terrigenous matter characterized by high REE concentrations with distinctly non rate them disproportionately (Bau et al., 1996; Bau and Koschinsky, seawater like patterns (Goldstein and Jacobsen, 1988; Elderfield et al., 2009). Moreover, Bayon et al. (2004) demonstrated that conspicuous 1990); 2) Fe and Mn oxides, which incorporate REE disproportionately amounts of Fe Mn oxyhydroxides may be inherited by aeolian dust and (Bau et al., 1996) and are prone to early diagenetic modifications (Bayon "preformed oxides" carried by riverine particulates. Thus the REE et al., 2004); 3) phosphates, which have a high affinity for REE in signature of Fe Mn oxyhydroxides may vary substantially from the diagenetic fluids and in some cases show non uniform incorporation original seawater likeREE signature of authigenic precipitates. None (German and Elderfield, 1990; Byrne et al., 1996). theless, SRG limestones are characterized by $\mathrm{MnO}$ and $\mathrm{Fe}_{2} \mathrm{O}_{3}$ contents

We must also consider effects due to sample leaching procedure that below $0.2 \%$ and $0.8 \%$, respectively (Table 2 ). The leachate analyzed in this can bring into solution different phases of the paragenetic sequence. work is considered representing the carbonate fraction and loosely Samples 2 and 8 were leached both with hydrochloric and acetic acid to bound elements (see Bau and Koschinsky, 2009) containing only Mn and evaluate potential differences in the leaching behavior (Table 2). Fe hosted in carbonate and, therefore, it should carry only a small fraction Comparison between the two data sets (Table 2) reveals that many trace of the REE budget hosted by Fe and Mn oxides (cf. Rongemaille et al., elements (e.g. P, Sc, Ti, V, Cr) were more strongly leached by hy 2011). Total REE and Fe contents in SRG limestones do not correlate drochloric acid. To the contrary, REE were more efficiently leached by suggesting that the REE patterns of SRG limestones are representative of acetic acid. The analysis of sample 2 leached with acetic acid yielded an the carbonate fraction.

average increase of $18.5 \%$ for REE consistent with increases in $\mathrm{Sr}$
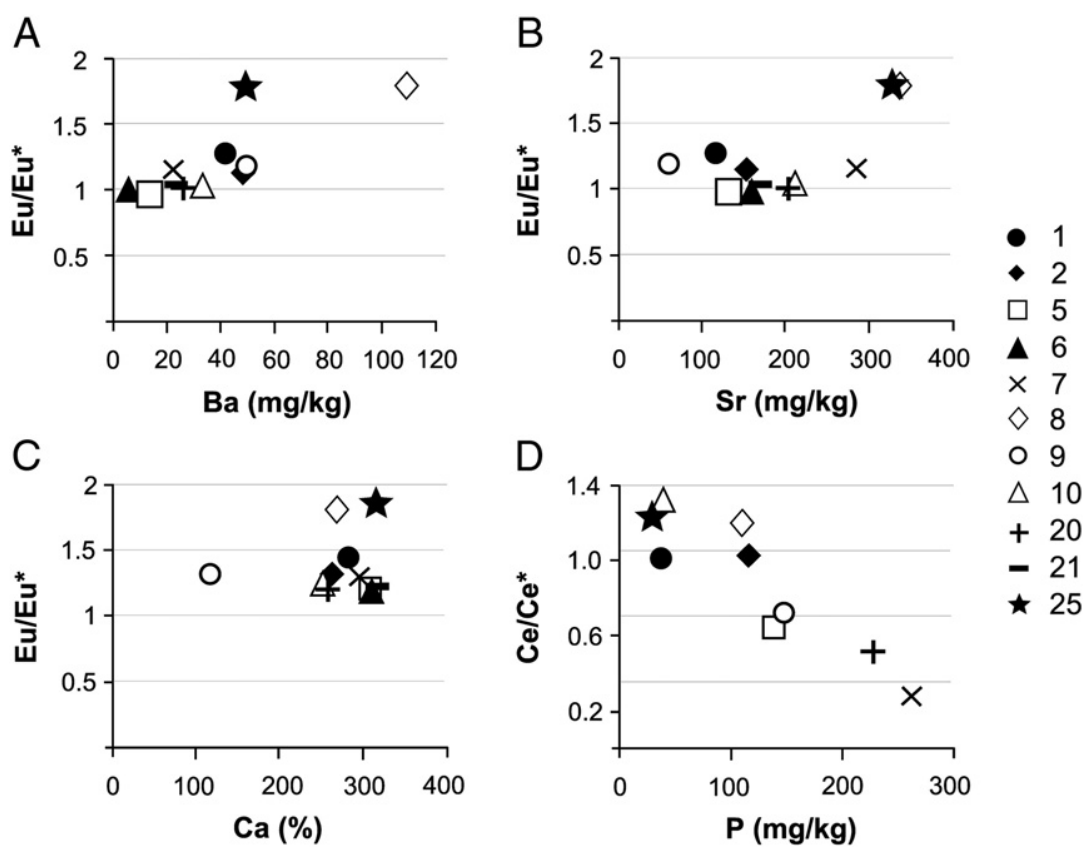

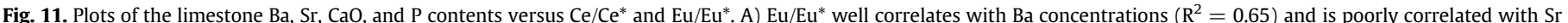

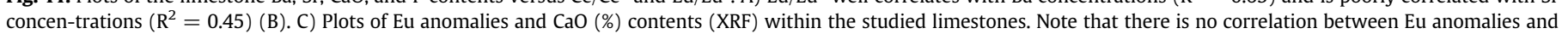
$\mathrm{CaO}$ contents. D) Negative correlation between $\mathrm{Ce}$ anomalies and $\mathrm{P}$ contents $\left(\mathrm{R}^{2}=0.82\right)$ within nine of the studied samples (see text for discussion). 
Phosphates also incorporate REE disproportionally and are prone to alteration during diagenesis (e.g., German and Elderfield, 1990; Reynard et al., 1999). Phosphorus contents correlate well with $\mathrm{Y} / \mathrm{Ho}$ ratios $\left(\mathrm{R}^{2}=\right.$ 0.74), whereas there is no correlation between P and $\Sigma$ REE (see Supplementary materials S3). Considering that those samples with high, superchondritic Y/Ho ratio are typically marine (Bolhar et al., 2004; Allwood et al., 2010), the positive correlation of $\mathrm{Y} / \mathrm{Ho}$ and $\mathrm{P}$ in the studied limestones suggests the association of $\mathrm{P}$ with marine precipitates.

\subsection{Trace elements and REE patterns}

The REE contents of SRG limestones vary systematically with facies. Variations are less pronounced in samples inferred to be part of the Kess Kess plumbing system. The variation of REE + Y patterns from different facies of the upper SRG suggests the preservation of the original signatures of the limestones that in case of incipient diagenesis would have been overprinted resulting in at least homogeneous REE patterns $(\S 5.1)$.

Besides variations of Ce and $\Sigma$ REE content the majority of samples show a seawater REE $+\mathrm{Y}$ pattern. The shale normalizedREE $+\mathrm{Y}$ pattern of modern seawater has typical key features: 1) strong LREEdepletion $\left(\mathrm{Pr}_{\mathrm{SN}} / \mathrm{Yb}_{\mathrm{SN}}\right.$ ranges between 0.2 and 0.4 ; Olivier and Boyet, 2006); 2) negative Ce and positive La anomalies (Fig. 10); 3) pos itive $\mathrm{Gd}$ anomaly $\left(\mathrm{Gd} / \mathrm{Gd}^{*}\right.$ of modern shallow seawater ranging be- tween 1.30 and 1.05 from the data in de Baar et al., 1985; Zhang and Nozaki, 1998 respectively); 4) super chondritic Y/Ho ratio (44 74, Bau, 1996).

The total REE contents of the SRG limestones reflect the relative abundance of micrite, cement and skeletal remains as obtained from point counting (Fig. 12). With the exclusion of two outliers (samples 6 and 21), samples with lower micrite contents also have lower $\Sigma$ REE contents. In our dataset the highest REE contents were detected in samples of trilobite floatstone and fossiliferous limestone (samples 5 and 10; ¿REE $17.78 \mathrm{mg} / \mathrm{kg}$ and $47.50 \mathrm{mg} / \mathrm{kg}$, respectively). These facies have a high amount of skeletal remains and micrite and are almost devoid of cement phases. Skeletal remains seem to have a marginal role in $\Sigma$ REE enrichment. Indeed, within the trilobite bearing limestone higher $\Sigma$ REE content was detected where the cement is less abundant. Therefore it can be inferred that micrite is the main REE carrier. The same trend has been described by Feng et al. (2009) from seep related carbonates. The authors argued that $\mathrm{REE}$ contents in micrite higher than in later carbonate phases suggests that diagenesis did not change the overall REE content.

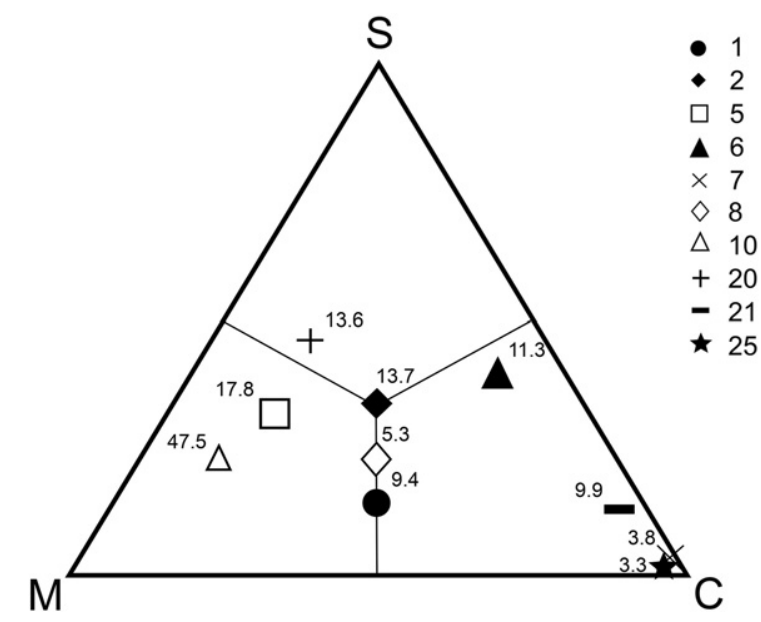

Fig. 12. Carbonatic phases and $\Sigma$ REE contents (in $\mathrm{mg} / \mathrm{kg}$ ) of the SRG limestones plotted in a ternary diagram. The three carbonatic endmembers are micrite $(M)$, cement $(C)$, and skeletal remains (S). Sample 9 is excluded due to its high clastic quartz content. The diagram shows that samples with high micrite content have high $\Sigma$ REE contents, whereas cement-rich samples show lower ZREE contents.

\subsubsection{Eu anomaly as an indicator for hydrothermal fluid circulation}

Bełka (1998) reported $\Sigma$ REE contents in Kess Kess mound micrite less $(\sim 15 \mathrm{mg} / \mathrm{kg})$ than micrite of inter mound crinoidal wackestones $(\sim 40 \mathrm{mg} / \mathrm{kg})$. The author also reported chondrite normalized negative Eu anomalies in samples from inter mounds areas and positive Eu anomalies in micrite of mounds and from neptunian dyke fills. Bełka (1998) argued that REE pattern variations indicate changes in circulating fluid chemistry and considered the low REE content and the positive $\mathrm{Eu}$ anomaly as consistent with precipitation from hydrothermal fluids. Although our data set and that proposed by Bełka (1998) are based on different assumptions, it seems clear that the data presented here show a scenario far more complicated than those proposed in the earlier literature.

The studied samples show patterns characteristic of diverse water chemistries. As REE patterns of seafloor hydrothermal fluids co vary with the chemical and physical conditions of fluid rock interaction, we must consider that SRG overlies a ca. 35 meter thick succession of basaltic rocks (Merzane Group basaltic breccia) which show a distinct positive Eu anomaly (see Jakubowicz et al., 2015). Hydrothermal fluids of relatively high temperatures emanating from oceanic crust show a LREE enriched pattern relative to mid ocean ridge basalt (MORB) and frequently show a positive $\mathrm{Eu}$ anomaly due to breakdown of plagioclase (e.g. Michard, 1989). Such fluids have higher concentrations of REE compared to seawater and have chondritic Y/Ho ratios (e.g. Klinkhammer et al., 1994a; Bau and Dulski, 1999). Guy et al. (1999) re ported that interstitial fluids of Mururoa basalts were enriched in Eu under reducing conditions. Mitra (1994) suggested that hydrothermal input to the oceans could lead to a net depletion of total REE in seawater as a consequence of scavenging by Fe oxyhydroxides. As discussed above $\mathrm{Fe}_{2} \mathrm{O}_{3}$ contents within the eleven carbonates processed for REE analyses are always lower than 1\% (Table 2); nevertheless massive oc currences of Fe oxyhydroxides were reported from the upper part of the SRG (i.e. vents in Table 1). Although formation of these massive Fe oxyhydroxide deposits might suggests a local scavenging of REE from seawater due to adsorption onto the Feoxyhydroxides, $\Sigma$ REE content in sample 12 , for instance is consistent with average $\Sigma$ REE content of the studied limestones (Table 4). Sample from quartz veinlets from the same facies has a very low $\Sigma$ REE content $(1.20 \mathrm{mg} / \mathrm{kg})$ coupled with high Eu anomaly (>4), suggesting likely precipitation of this silica phase from hydrothermal fluids that interacted with basalt. Neverthe less, the time of formation of these veinlets is still unclear and may postdate Kess Kess mound formation.

Among SRG carbonates only samples 8 and 25 (Table 4) show a consistent Eu anomaly (Fig. 11), which can, but not unambiguously (see discussion below), be related to hydrothermal fluid circulation (Klinkhammer et al., 1983, 1994a; McLennan, 1989; Derry and Jacobsen, 1990). Hydrothermal fluids are commonly enriched in Ba (e.g. Olivarez and Owen, 1989; Klinkhammer et al., 1994b; Douville et al., 1999) and Ba rich deposits normally typify hydrothermal vents (e.g. Church, 1979; Douville et al., 1999). Within SRG limestones Eu anomaly correlates with Ba contents $\left(\mathrm{R}^{2}=0.65\right)$, which is highly concentrated in sample 8 (Table 2). Therefore, we cannot exclude that the positive Eu anomaly within these samples might have been inherited from hydrothermal fluid with temperature ranging between $4050{ }^{\circ} \mathrm{C}$ and $120^{\circ} \mathrm{C}$ (Bełka, 1998; Mounji et al, 1998; Franchi et al., 2014).

\subsubsection{Ce anomaly as an indicator for palaeoredox conditions}

In oxygenated environments Ce occurs as $\mathrm{Ce}(\mathrm{IV})$ that can easily be scavenged by Fe oxyhydroxides (e.g. Derry and Jacobsen, 1990) with consequent negative $\mathrm{Ce}$ anomaly of seawater and derived marine precipitate. The value of the Ce anomaly, therefore, is thought to be indicative of seawater redox state (Haley et al., 2004 and citations therein): Ce anomaly around 0.20 .4 suggests an oxygenated oceanic conditions, whereas a positive Ce anomaly suggests anoxic conditions (Olivier and Boyet, 2006; Oliveri et al., 2010: Hu et al., 2014) or the presence of $\mathrm{Ce}$ enriched $\mathrm{Fe}$ oxyhydroxides or particulate matter. 
Nevertheless, the interpretation of Ce anomaly in marine precipitates is complicated by possible La anomalies, as anomalous Ce abundances may result from anomalous La enrichment. Fig. 10 shows that for most of the SRG samples $\mathrm{Ce} / \mathrm{Ce}^{*}$ ratios below unity result from positive La anomaly as suggested by $\mathrm{Pr} / \mathrm{Pr}^{*}$ ratio close to 1 . The SRG limestones are grouped by different values of Ce anomaly (Fig. 10): 1) negative Ce anomaly (samples 5, 7, 9 and 20), 2) lack of consistent Ce anomaly (samples 1, 2, 8, 10 and 25) and 3) strong positive Ce anomaly (samples 6 and 21). As shown in Fig. 10 only the first group (samples 5, 7, 9 and 20) has Ce and La anomalies consistent with modern seawater (e.g. Nothdurft et al., 2004). On the other hand, the negative Ce anomaly detected in samples from mound facies may be the result of high pore water alkalinity in the presence of organic matter (e.g. Kim et al., 2012; Hu et al., 2014). The metabolic activity of microbial consortia within organic matter rich sediments increases pore water alkalinity triggering the precipitation of authigenic carbonates (e.g. Chafetz and Buczynski, 1992; Monty, 1995). In this scenario the preferential uptake of dissolved $\mathrm{Ce}(\mathrm{IV})$ on organic compounds induces a negative $\mathrm{Ce}$ anom aly in the pore fluids (Pourret et al., 2008; Kim et al., 2012; Hu et al., 2014). We did not evaluate the amount of organic matter within our samples, but Raman analyses revealed the presence of disordered carbonaceous matter (Fig. 5). Guido et al. (2012) reported high organic matter contents within laminated micrite from mound facies. The authors found organic compounds possibly recording microbial and/or archea communities. A preferential Ce uptake by microbial communi ties seems therefore feasible for the mound facies. Other authors observed that even intermittent oxygenation, coupled with microbial activity, might result in uptake of $\mathrm{Ce}(\mathrm{IV})$ and consequent negative Ce anomaly (Himmler et al., 2010; Kim et al., 2012; Hu et al., 2014).

Trace elements such as $U$ and Mo are commonly used to better constrain palaeoredox conditions. Uranium (and Mo) exhibits conser vative behavior under oxic conditions, being present mainly as $\mathrm{U}(\mathrm{VI})$, whereas it is enriched (as insoluble U(IV) in authigenic mineral phases, particularly iron sulfides, under reducing conditions (Tribovillard et al., 2006; Hu et al., 2014). We should therefore consider that, a leaching process via dilute acid is probably unable to bring into solution $U$ and Mo hosted within authigenic iron sulfides. $\mathrm{Hu}$ et al. (2014) reported that Mo concentration within carbonate phases (weak acid leaching) is $1 / 20$ of the concentration detected in the bulk sample (strong acid leaching). Here we compare trace element concentrations obtained from the carbonate phases by weak acid leaching of different carbonatic facies avoiding any consideration on the total content of trace elements in the bulk rock. In our data set $\mathrm{U}$ concentrations show wide variations (between $0.14 \mathrm{mg} / \mathrm{kg}$ and $18.79 \mathrm{mg} / \mathrm{kg}$ ) suggesting changes in redox conditions. Several samples show higher $U$ contents (up to 3 orders of magnitude in samples 1,8 and 10) which might reflect deposition of carbonates under anoxic conditions (Hu et al., 2014).

Additional evidence of variable redox conditions in fluid pathways (e.g. veins) is the Ce enrichment within trilobite bearing limestones. Fig. 10 shows that the trilobite bearing facies recorded two possible redox conditions: oxic condition during deposition of trilobite floatstone (sample 5); anoxic condition during deposition of trilobite rudstone (sample 6). The variation of these geochemical parameters may be related to increase of alkalinity or seawater mixing, probably with hydrothermal fluids (e.g. McLennan, 1989). Such fluids are commonly characterized by depletion in dissolved oxygen due to reactions with reduced wall rock components (e.g. Tunnicliffe et al., 2003). Fluid circulation through the trilobite bearing facies is also suggested by patchy $\alpha$ quartz (Figs. 5A, B and 6A) and goethite (Fig. 5B) replacing micrite and trilobite shells (Franchi et al., 2014). Quartz is a common precipitate during cooling of fluids supersaturated with respect to Si after interaction with volcanic rocks (Ellis and McMahon, 1977). Hydro thermal water flux from volcanic rocks of the Merzane Group flowing through SRG to the trilobite bearing facies could have been responsible for the partial dissolution of low magnesium calcite (LMC) and conse quent deposition of $\alpha$ quartz (Franchi et al., 2014). The $\alpha$ quartz is the thermodynamically stable phase of silica formation and recrystallization at hydrothermal vents (Rodgers et al., 2004). Sample of microcrystalline $\alpha$ quartz revealed peloidal and botryoidal fabrics (Fig. 5D) typical of chalcedony (see Rodgers et al., 2004) described from active siliceous hot springs (Fig. 6E, in Guidry and Chafetz, 2003).

A separate discussion is required for pisolite bearinginter mound facies sample 9. Pisolites form within the vadose zone of soils and shallow marine settings where calcite cements precipitate around clastic nuclei trapped in sediments. Sample 9 displays a strong MREEenrichment $\left(\operatorname{Pr}_{\mathrm{SN}} / \mathrm{Dy}_{\mathrm{SN}}=1.8\right)$, which is a typical characteristic of pore water of iron reduction zone (Haley et al., 2004; Hu et al., 2014). This evidence, coupled with a slight negative Ce anomaly, points to precipitation of this facies under suboxic conditions (e.g. Kim et al., 2012; Hu et al., 2014). This facies may therefore be related to diagenetic alteration of host carbonate deposits in the vadose zone during partial exposure (e.g. Fu et al., 2004). Evidence for partial exposure has been recorded in the area starting from the Famennian (Bełka, 1998). Brachert et al.(1992) reported karstic structures in the upper part of the SRG. Never theless, the influence of vadose water should be restricted to the pisolite facies in the upper strata of the group, as suggested by the depletion in LREE and MREE of carbonates from mound and inter mound areas appearing inconsistent with an early meteoric (phreatic and/or vadose) diagenesis (e.g. Moldovanyi et al., 1990).

\subsubsection{Y/Ho ratio: consistency of a seawater signature}

$\mathrm{Y} / \mathrm{Ho}$ ratio gives other important information for seawater characterization. Yttrium is commonly considered as a chemical twin of Ho (e.g. Bau, 1996) and thus expected to exhibit similar geochemical behavior in seawater (e.g., Zhang et al., 1994; Nozaki et al., 1997). The geochemical twins $\mathrm{Y}$ and Ho remain tightly coupled in many geochem ical processes, leading to the maintenance of the chondritic Y/Ho ratio $(\sim 2628)$ in common igneous rocks and epiclastic sediments (Bau, 1996, and references therein). In marked contrast, however, aqueous fluids and their precipitates are often characterized by super chondritic $\mathrm{Y} / \mathrm{Ho}$ ratio (up to 90), typical of modern seawater (Zhang et al., 1994; Bau, 1996; Bau et al., 1997; Nozaki et al., 1997). Strong positive Y anomaly in the SRG samples resulted in a super chondritic Y/Ho ratio (up to 87) within samples 5, 6, 7, 11, 20, and 21 (Table 4). Whereas samples 1, 2, 8, 9, 10, 12, and 25 show Y/Ho ratio lower than 50 (Table 4), closer to chondritic values. With the exclusion of trilobite rudstone and vein filling (samples 6 and 21, respectively), there is a clear linear correlation between $\mathrm{Y} / \mathrm{Ho}$ and La anomaly $\left(\mathrm{R}^{2}=0.93\right.$ )(Fig. 13). Samples with the highest $\mathrm{Y} / \mathrm{Ho}$ also have the strongest La anomaly (Fig. 13) and show typical seawater signatures (e.g. Bau, 1996; Webb and Kamber, 2000; Allwood et al., 2010). Less pronounced marine signals within samples 6, 8, and 21 were probably produced by sea water mixing with hydrothermal water. Mixing of seawater with hydrothermal fluids is consistent with La excess (e.g. Klinkhammer et al., 1994a; Kamber and Webb, 2001) found, for instance, in samples 6 and 21 (Table 4). This mixing occurred in peculiar facies, as suggested by the presence of $\mathrm{Ce}$ and Eu anomalies in samples from the plumbing

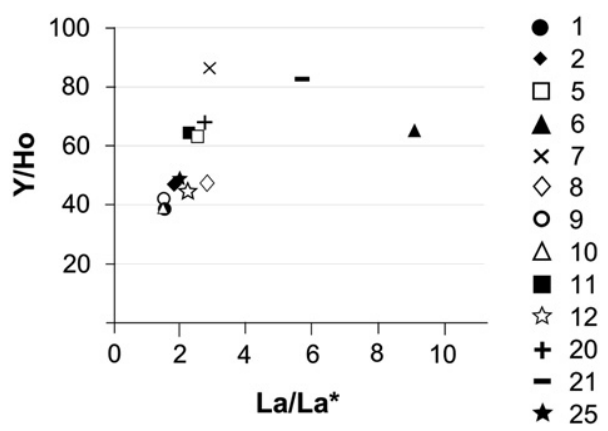

Fig. 13. Plot of $Y /$ Ho ratios versus La anomaly: $R^{2}=0.58$ without sample $6 ; R^{2}=0.64$ without samples 6 and 21 . 
system (samples 6 and 21) and layered micrite (sample 8), which is consistent with poorly oxygenated (e.g. Hu et al., 2014) and warm fluid circulation through volcanic rocks (Michard, 1989).

\subsubsection{Fluid circulation and seawater mixing}

Stable isotopic composition of SRG carbonates may provide better constraints for microbial activity and water temperature during carbon ate formation. The $\delta^{13} \mathrm{C}$ and $\delta^{18} \mathrm{O}$ values reported by Franchi et al. (2014) are shown in Table 4. Carbonates from layered micrite and vein filling (samples 8 and 25 , respectively) show strong ${ }^{13} \mathrm{C}$ depletion revealing possible seawater mixing with venting fluids and linked microbial activity (see also Cavalazzi et al., 2007). Alternatively, as suggested by Franchi et al. (2014), the ${ }^{13} \mathrm{C}$ depletion in some of the SRG samples may have been inherited by the consumption of sedimentary organic matter $\left(\delta^{13} \mathrm{C}=-20 \%\right.$, see Aloisi et al., 2002) by sulfate reducing bacteria (e.g. Ritger et al., 1987). Nevertheless these samples show the lowest REE contents in the data set (Table 4). This characteristic of SRG carbonate apparently rules out microbial activity as a REE enrich ment factor. The slightly negative $\delta^{13} \mathrm{C}$ values of SRG samples could be also related to the contribution of hydrothermal crustal $\mathrm{CO}_{2}$ (with an average $\delta^{13} \mathrm{C}=-7 \%$; Konno et al., 2006; Franchi et al., 2014) and/or abiotic methane (Bełka, 1998; Cavalazzi et al., 2007). The peculiar signature of some of the SRG carbonates may therefore be independent from microbial activity and may have been inherited by venting fluids. The enrichment of REE within the mounds limestones is probably due to a kinetic effect, indeed the slow precipitation of micrite may lead to the adsorption of large quantities of REE (e.g. Webb et al., 2009; Jakubowicz et al., 2015).

High temperature fluids interacting with basaltic rocks become enriched in $\mathrm{Eu}(\mathrm{II})$ (e.g. Bau and Dulski, 1999). Accordingly, samples from the SRG plumbing system (e.g. sample 25) show the highest Eu anomaly coupled with lower $\Sigma$ REE contents, rather typical for hydrothermal fluids (Mitra, 1994), positive Ce anomaly and negative $\delta^{18} \mathrm{O}$ (Table 4). Layered micrite (sample 8 ), showing $\delta^{18} \mathrm{O}=-10.1 \%$, low ¿REE content and positive Eu anomaly (Table 4), is probably part of the plumbing system branching within the mound buildups.

Once the hot venting fluids have reached the surface the mixing with large volumes of sea water gave rise to the disappearance of the $\mathrm{Eu}$ anomaly and $\mathrm{Eu}(\mathrm{II})$ has been rapidly oxidized to $\mathrm{Eu}(\mathrm{III})$. Cement forma tion in this case has been probably triggered by gradual anaerobic consumption of organic matter as suggested by the slightly positive $\delta^{13} \mathrm{C}$ values and Ce anomaly (Table 4 ).

\subsection{REE pattern and Devonian seawater}

To make full use of ancient limestones as proxies for ancient seawater requires knowledge of the partition coefficients between seawater and limestone (Elderfield et al., 1990; Webb and Kamber, 2000; Tanaka and Kawabe, 2006). Although the preservation of seawater REE signatures in marine precipitates has been widely documented (Webb and Kamber, 2000; Kamber and Webb, 2001; Nothdurft et al., 2004), and partition coefficients are equivalent across the whole range of investigated Holocene limestones (Webb and Kamber, 2000), we cannot predict exact partition coefficients for different ancient limestones. Such partition coefficients cannot be determined directly, as ancient seawaters are not preserved. Hence, reasonable partition coefficients must be hypothesized on the basis of experimental work and analogy with modern proxies (Webb and Kamber, 2000; Nothdurft et al., 2004; Bau and Koschinsky, 2009; Della Porta et al., 2015). Although partition behavior may have been somewhat different in different carbonates (Webb and Kamber, 2000; Della Porta et al., 2015), evidence from ancient limestones supports the use of modern precipitates (i.e. microbialite) partition coefficients as a model for ancient samples (Webb and Kamber, 2000; Nothdurft et al., 2004). Webb and Kamber (2000) calculated consistent mean partition coefficients ( $\left.D_{\mathrm{REE}}=295.6\right)$ across the entire mass range of Holocene microbialite REEs (SD = 30.2; excluding Ce):

$\mathrm{D}_{\mathrm{REE}_{\text {CARBONATE/SEAWATER }}}\left(\frac{\text { REE }_{\text {CARBONATE }}}{R E E_{\text {SEAWATER }}}\right) *\left(\frac{C a_{\text {SEAWATER }}}{C a_{\text {CARBONATE }}}\right)$.

We calculated the REE pattern for Devonian seawater following the method given by Tanaka and Kawabe (2006), starting from SRG lime stone REE composition and standard water conditions (Fig. 14).

As shown in Fig. 13 studied limestones are clustered along the $\mathrm{Y} / \mathrm{Ho}$ La anomaly trend line. Average values of samples with strong La Y/Ho correlation (samples 7 and 20), from mound facies (samples 2, 8, 10) as well as representing outliers, such as samples from trilobite rudstone and vein filling (plumbing system), were used for seawater calculations. The shale normalized Early Devonian seawater patterns were then compared with data from Zhang and Nozaki (1996) for open Pacific Ocean seawater (Fig. 14). Although REE contents estimated for Early Devonian seawater are higher than modern seawater contents (i.e. Zhang and Nozaki, 1996), several considerations can be drawn. The higher contents in Devonian seawater might be due to diagenetic disso lution of organic matter and/or due to microbial activity, as well as kinetic effects. Microbial communities through their extracellular polysaccharides (EPS) might have increased REE abundances in SRG limestones (e.g. Webb and Kamber, 2000; Nothdurft et al., 2004) with the consequent overestimation of REE abundances in seawater.

Besides the high $\Sigma$ REE contents the Early Devonian seawater REE pattern derived from normal marine samples is consistent with the modern seawater showing strong depletion of LREE, super chondritic $\mathrm{Y} / \mathrm{Ho}$ ratio, positive Gd anomaly (average 1.4) and negative $\mathrm{Ce}$ anomaly. The seawater pattern obtained from mound facies limestones is characterized by less strong LREE depletion $\left(\mathrm{Pr}_{\mathrm{SN}} / \mathrm{Yb}_{\mathrm{SN}}\right.$ $=0.5$ ) and smaller positive $\mathrm{Ce}$ and Eu anomalies resulting in a less fractionated REE pattern (Fig. 14).

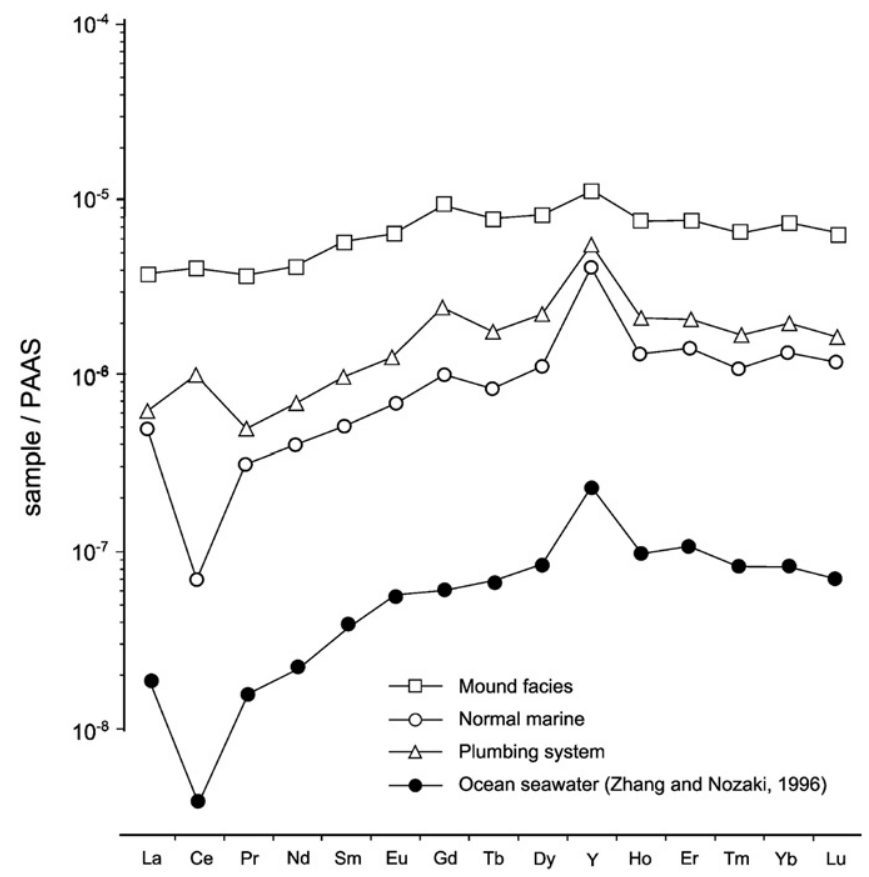

Fig. 14. PAAS-normalized REE abundances patterns for Early Devonian seawater estimated from the REE patterns of different SRG facies. The "plumbing system" pattern comes from the average composition of samples 6 and 21. We used the partition coefficients given by Tanaka and Kawabe (2006); seawater conditions: $\mathrm{pH}=8.2$; $\left[\mathrm{CO}_{2}\right]_{\text {TOT }}=0.00235$; salinity $=35 \%$. REE pattern of modern off-shore surface (filled circles) seawater by Zhang and Nozaki (1996) are plotted for comparison (average of 0 , 9, and $30 \mathrm{~m}$ depth samples from station SA-5, East Caroline Basin, data from Zhang and Nozaki, 1996; see also Webb and Kamber, 2000). 
Early Devonian seawater REE patterns derived from the plumbing system samples (samples 6 and 21) show similar trends. This implies precipitation of these facies from the same water which is consistent with the hypothesis of a common plumbing system within mound facies. Early Devonian seawater REE compositions obtained from the plumbing system show a seawater likeREE pattern and a strong positive Ce anomaly (up to 3.8 ). This could imply that the waters flowing through the plumbing system were not sufficiently oxidizing to allow formation of $\mathrm{Ce}$ (IV) species which is more reactive with particle surfaces from which it is less efficiently desorbed (Kamber and Webb, 2001). Within these facies seawater mixed with fluids with different chemical composition and probably depleted in $\mathrm{O}_{2}$ contents.

\section{Concluding remarks}

This work focused on detailed analyses of trace element variations and REE patterns in limestones and associated chemical precipitates from the Early Devonian Kess Kess mounds. The REE patterns, as well as redox sensitive trace element distributions, have been affected by mixing of seawater with warm hydrothermal fluids during the formation of the Kess Kess mounds. In combination with previous sedimento logical and geochemical data, the following conclusions can be drawn:

1. The SRG limestones show REE + Y patterns characterized by LREE depletion, super chondritic $\mathrm{Y} / \mathrm{Ho}$ ratio, and positive La and Gd anomalies. Although this pattern is in close agreement with the modern seawater REE pattern, we found that the composition of SRG lime stone varies with facies recording fluctuation of redox conditions.

2. The main variations in $\Sigma$ REE contents are consistent with a diverse array of petrographic features. Our results underscore that, in the SRG limestones, micrite is the main REE carrier probably due to kinetic effects.

3. The influence of low oxygenated warm fluids was probably restricted to certain facies. Quartz from veinlets that breach the vent host rocks in the upper part of the SRG exhibits a large positive Eu anom aly and low overall $\Sigma$ REE contents. Other samples from the SRG plumbing system show large positive Eu anomaly coupled with low $\Sigma$ REE contents, positive Ce anomaly and negative $\delta^{18} \mathrm{O}$. The presence of a positive Ce anomaly and less pronounced marine signals (i.e. La excess) in trilobite rudstone and vein fills (mound facies) validates the model promoting mixing of seawater with poorly oxygenated fluids within the plumbing system.

4. Positive Eu anomalies recorded by samples of the plumbing system, coupled with low $\Sigma$ REE contents and negative $\delta^{18} \mathrm{O}$ values, is sugges tive of seawater mixing with hydrothermal fluids that interacted with underlying Merzane Group basaltic rocks.

5. The lack of a Eu anomaly and the modern seawater like negative Ce anomaly of the mound limestones suggests deposition within well oxygenated seawater. Hydrothermal fluids reaching the surface mixed with large volumes of seawater losing their original Eu anom aly. The high ZREE content within mound carbonates is probably due to kinetic effects and/or to the microbial mediated dissolution of organic matter during the early diagenesis.

6. Microbial consumption of organic matter, under anoxic conditions, could have locally led to $\mathrm{Ce}$ fractionation, giving rise to a positive $\mathrm{Ce}$ anomaly. The increase of pore water alkalinity due to metabolic activity of microbial consortia within organic matter rich sediments triggered the precipitation of authigenic carbonates. This process likely occurred within the trilobite bearing limestones.

7. The data set obtained from SRG limestones allowed to estimate the REE pattern of Early Devonian seawater, which is similar to the modern seawater REE pattern. Similar calculations using REE abundances of plumbing system facies revealed a strong positive Ce anomaly consistent with poorly oxygenated fluids. Supplementary data to this article can be found online at http://dx.

doi.org/10.1016/j.chemgeo.2015.05.006.

\section{Acknowledgments}

This research was supported by the MIUR (PRIN 2010) program "Comparative geomicrobiology of the mounds in the Moroccan Sahara: implications for Mars astrobiology" (to RB). Thanks are due to Christian Reinke and Eve Fisher (University of Johannesburg) for their technical assistance, and to Frantz Gerard Ossa Ossa (University of Johannesburg) for XRF analyses. The authors acknowledge two anonymous reviewers and the journal Editor Michael Böttcher for constructive comments and suggestions which greatly improved the manuscript. FF would like to thank Marco Taviani and Paolo Montagna (CNR ISMAR USOBologna) for precious advice. This is ISMAR CNR, Bologna, scientific contribution n. 1818.

\section{References}

Aitken, S.A., Collom, C.J., Henderson, C.M., Johnston, P.A., 2002. Stratigraphy, paleoecology, and origin of Lower Devonian (Emsian) carbonate mud buildups, Hamar Laghdad, eastern Anti-Atlas, Morocco, Africa. Bull. Can. Petrol. Geol. 50, 217-243.

Allwood, A.C., Kamber, B.S., Walter, M.R., Burch, I.W., Kanik, I., 2010. Trace elements record depositional history of an Early Archean stromatolitic carbonate platform. Chem. Geol. 270, 148-163.

Aloisi, G., Pierre, C., Rouchy, J.-M., FauGèresc, J.-C., 2002. Isotopic evidence of methane-related diagenesis in the mud volcanic sediments of the Barbados Accretionary Prism. Cont. Shelf Res. 22, 2355-2372.

Banner, J.L., Hanson, G.N., Meyers, W.J., 1988. Rare earth element and Nd isotopic variations in regionally extensive dolomites from the Burlington-Keokuk Formation (Mississippian): implications for REE mobility during carbonate diagenesis. J. Sediment. Petrol. 58, 415-432.

Bau, M., 1996. Controls on the fractionation of isovalent trace elements in magmatic and aqueous systems: evidence from $\mathrm{Y} / \mathrm{Ho}, \mathrm{Zr} / \mathrm{Hf}$, and lanthanide tetrad effect. Contrib.

Mineral. Petrol. 123, 323-333.

Bau, M., Dulski, P., 1996. Distribution of yttrium and rare-earth elements in the Penge and Kuruman iron-formations, Transvaal Supergroup, South Africa. Precambrian Res. 79, 37-55.

Bau, M., Dulski, P., 1999. Comparing yttrium and rare earths in hydrothermal fluids from the Mid-Atlantic Ridge: implications for $Y$ and REE behaviour during near-vent mixing and for the Y/Ho ratio of Proterozoic seawater. Chem. Geol. 155, 77-90.

Bau, M., Koschinsky, A., 2009. Oxidative scavenging of cerium on hydrous Fe oxide: evidence from the distribution of rare earth elements and yttrium between Fe oxides and Mn oxides in hydrogenetic ferromanganese crusts. Geochem. J. 43, 37-47.

Bau, M., Koschinsky, A., Dulski, P., Hein, J.R., 1996. Comparison of the partitioning behaviours of yttrium, rare earth elements, and titanium between hydrogenetic marine ferromanganese crusts and seawater. Geochim. Cosmochim. Acta 60, 1709-1725.

Bau, M., Möller, P., Dulski, P., 1997. Yttrium and lanthanides in eastern Mediterranean seawater and their fractionation during redox-cycling. Mar. Chem. 56, 123-131. Bayon, G., German, C.R., Burton, K.W., Nesbitt, R.W., Rogers, N., 2004. Sedimentary Fe-Mn oxyhydroxides as paleoceanographic archives and the role of aeolian flux in regulat-ing oceanic dissolved REE. Earth Planet. Sci. Lett. 224, 477-492.

Bełka, Z., 1998. Early Devonian Kess-Kess carbonate mud mounds of the eastern Anti-Atlas (Morocco), and their relation to submarine hydrothermal venting. J. Sediment. Res. 68, 368-377.

Bełka, Z., Berkowski, B., 2005. Discovery of thermophilic corals in an ancient hydrothermal vent community, Devonian, Morocco. Acta Geol. Pol. 55, 1-7.

Berkowski, B., 2004. Monospecific rugosan assemblage from the Emsian hydrothermal vents of Morocco. Acta Geol. Pol. 49, 75-84.

Berkowski, B., 2006. Vent and mound rugose coral associations from the Middle Devonian of Hamar Laghdad (Anti-Atlas, Morocco). Geobios 39, 155-170.

Berkowski, B., 2008. Emsian deep-water Rugosa assemblages of Hamar Laghdad (Devonian, Anti-Atlas, Morocco). Palaeontogr. Abt. A284, 17-68.

Berkowski, B., 2012. Life strategies and function of dissepiments in rugose coral Catactotoechus instabilis from the Lower Devonian of Morocco. Acta Palaeontol. Pol. 57, 391-400.

Berkowski, B., Klug, C., 2012. Lucky rugose corals on crinoid stems: unusual examples of subepidermal epizoans from the Devonian of Morocco. Lethaia 45, 24-33.

Bertram, C.J., Elderfield, H., 1993. The geochemical balance of the rare earth elements and neodymium isotopes in the oceans. Geochim. Cosmochim. Acta 57, 1957-1986. Bolhar, R., Kamber, B.S., Moorbath, S., Fedo, C.M., Whitehouse, M.J., 2004. Characterisation of early Archaean chemical sediments by trace element signatures. Earth Planet. Sci. Lett. 222, 43-60.

Brachert, T.C., Buggisch, W., Flügel, E., Hüssner, H.M., Joachimski, M.M., Tourneur, F., Walliser, O.H., 1992. Controls of mud mound formation: the Early Devonian KessKess carbonates of the Hamar Laghdad, Antiatlas, Morocco. Geol. Rundsch. 81, 11-44.

Byrne, R.H., Liu, X., Schijf, J., 1996. The influence of phosphate coprecipitation on rare earth element distributions in natural waters. Geochim. Cosmochim. Acta 60, 3341-3346. Cavalazzi, B., Barbieri, R., Ori, G.G., 2007. Chemosynthetic microbialites in the Devonian carbonate mounds of Hamar Laghdad (Anti-Atlas, Morocco). Sediment. Geol. 200, 73-88.

Cavalazzi, B., Barbieri, R., Cady, S.L., George, A.D., Gennaro, S., Lui, A., Westall, F., Rossi, A.P., Ori, G.G., Taj-Heddine, K., 2012. Iron-framboids in the hydrocarbon-related Middle Devonian Hollard Mound of the Anti-Atlas mountain range in Morocco: evidence of potential microbial biosignatures. Sediment. Geol. 263-264, 183-193. 
Chafetz, H.S., Buczynski, C., 1992. Bacterially induced lithification of microbial mats. Palaios 7, 277-293.

Church, T.M., 1979. Marine barite. In: Burns, R.G. (Ed.), Marine Minerals. Miner. Soc. Am. Rev. Miner. 6, pp. 170-210.

De Baar, H.J.W., Bacon, M.P., Brewer, P.G., Bruland, K.W., 1985. Rare earth elements in the Pacific and Atlantic oceans. Geochim. Cosmochim. Acta 49, 1943-1959.

De Baar, H.J.W., Schijf, J., Byrne, R.H., 1991. Solution chemistry of the rare earth elements in seawater. Eur. J. Solid State Inorg. Chem. 28, 357-373.

Della Porta, G., Webb, G.E., McDonald, I., 2015. REE patterns of microbial carbonate and cements from Sinemurian (Lower Jurassic) siliceous sponge mounds (Djebel Bou

Dahar, High Atlas, Morocco). Chem. Geol. 400, 65-86.

Derry, L.A., Jacobsen, S.B., 1990. The chemical evolution of Precambrian seawater: Evidence from REEs in banded iron formations. Geochim. Cosmochim. Acta 54, 2965-2977.

Douville, E., Bienvenu, P., Charlou, J.L., Donval, J.P., Fouquet, Y., Appriou, P., Gamo, T., 1999. Yttrium and rare earth elements in fluids from various deep-sea hydrothermal

systems. Geochim. Cosmochim. Acta 63, 627-643.

Downs, R.T., 2006. The RRUFF Project: an Integrated Study of the Chemistry, Crystallography, Raman and Infrared Spectroscopy of Minerals. Program and Abstracts of the 19th General Meeting of the International Mineralogical Association in Kobe, Japan, pp. 03-13 (O).

Elderfield, $H$. 1988. The oceanic chemistry of the rare-earth elements. Philos. Trans. $R$. Soc. Lond. 325, 105-106.

Elderfield, H., Upstill-Goddard, R., Sholkovitz, E.R., 1990. The rare earth elements in

riversstuaries and coastal sea waters: processes affecting crustal input of elements to the ocean and their significance to the composition of sea water. Geochim. Cosmochim. Acta 54, 971-991.

Ellis, E.J., McMahon, W.A.J., 1977. Chemistry and Geothermal System. Academic Press, New York, p. 392

Feng, D., Chen, D., Peckmann, J., 2009. Rare earth elements in seep carbonates as tracers of variable redox conditions at ancient hydrocarbon seeps. Terra Nova 21, 49-56. Franch F., Cavalazzi, B., Pierre, C., Barbieri, R., 2014. New evidences of hydrothermal fluids circulation at the Devonian Kess Kess mounds, Hamar Laghdad (eastern Anti-

Atlas, Morocco). Geol. J. http://dx.doi.org/10.1002/gj.2582.

Fu, Q., Qing, H., Bergman, K.M., 2004. Dolomitized calcrete in the Middle Devonian Winnipegosis carbonate mounds, subsurface of south-central Saskatchewan, Canada. Sediment. Geol. 168, 49-69.

German, C.R., Elderfield, H., 1990. Application of the Ce anomaly as a paleoredox indicator: the ground rules. Paleoceanography 5, 823-833.

Goldstein, S.J., Jacobsen, S.B., 1988. Rare earth elements in river waters. Earth Planet. Sci. Lett. 89, 35-47.

Greaves, M.J., Elderfield, H., Sholkovitz, E.R., 1999. Aeolian sources of rare earth elements to the Western Pacific Ocean. Mar. Chem. 68, 31-38.

Guido, A., Mastandrea, A., Demasi, F., Tosti, F., Russo, F., 2012. Organic matter remains in the laminated microfabrics of the Kess-Kess mounds (Hamar Laghdad, Lower Devo-

nian, Morocco). Sediment. Geol. 263-264, 194-201.

Guidry, S.A., Chafetz, H.S., 2003. Anatomy of siliceous hot springs: examples from Yellowstone National Park, Wyoming, USA. Sediment. Geol. 157, 71-106.

Guy, C., Daux, V., Schott, J., 1999. Behaviour of rare earth elements during seawater/basalt interactions in the Mururoa Massif. Chem. Geol. 158, 21-35.

Haley, B.A., Klinkhammer, G.P., McManus, J., 2004. Rare earth elements in pore waters of marine sediments. Geochim. Cosmochim. Acta 68, 1265-1279.

Himmler, T., Bach, W., Bohrmann, G., Peckmann, J., 2010. Rare earth elements in authigenic methane-seep carbonates as tracers for fluid composition during early

diagenesis. Chem. Geol. 277, 126-136.

Hollard, H., 1981. Principaux caractères des formations dévoniennes de l'Anti-Atlas. Notes Mém. Serv. Géol. Maroc. 42, 15-22.

Hu, Y., Feng, D., Peckmann, J., Roberts, H.H., Chen, D., 2014. New insights into cerium anomalies and mechanisms of trace metal enrichment in authigenic carbonate

from hydrocarbon seeps. Chem. Geol. 381, 55-66.

Jakubowicz, M., Berkowski, B., Betka, Z., 2013. Devonian rugose coral 'Amplexus' and its relation to submarine fluid seepage. Palaeogeogr. Palaeoclimatol. 386, 180-193.

Jakubowicz, M., Dopieralska, J., Bełka, Z., 2015. Tracing the composition and origin of

fluids at an ancient hydrocarbon seep (Hollard Mound, Middle Devonian, Morocco): a Nd,

REE and stable isotope study. Geochim. Cosmochim. Acta. http:// dx.doi.org/10.1016/j.gca.2015.02.027

Joachimski, M.M., Buggisch, W., Mounji, D., Bourque, P.-A., Savard, M.M., 1999. Hydrothermal origin of Devonian conical mounds (Kess-Kess) of Hamar Laghdad Ridge, Anti-

Atlas, Morocco: comment and reply. Geology 27, 863-864.

Johannesson, K.H., Hawkins Jr., D.L., Cortés, A., 2006. Do Archean chemical sediments record ancient seawater rare earth element patterns? Geochim. Cosmochim. Acta 70, 871-890.

Kamber, B.S., Webb, G.E., 2001. The geochemistry of Late Archaean microbial carbonate: implications for ocean chemistry and continental erosion history. Geochim.

Cosmochim. Acta 65, 2509-2525.

Kamber, B.S., Bolhar, R., Webb, G.E., 2004. Geochemistry of late Archaean stromatolites from Zimbabwe; evidence for microbial life in restricted epicontinental seas. Precam-brian Res. 132, 379-399.

Kaufmann, B., 1997. Diagenesis of Middle Devonian carbonate mud buildups of the Maïder Basin (eastern Anti-Atlas). J. Sediment. Res. A 67, 945-956.

Kawabe, I., Kitahara, Y., Naito, K., 1991. Non-chondritic yttrium/holmium ratio and lanthanide tetrad effect observed in pre-Cenozoic limestones. Geochem. J. 25, 31-44.

Kim, J.-H., Torres, M.E., Haley, B.A., Kastner, M., Pohlman, J.W., Riedel, M., Lee, Y.-J., 2012 The effect of diagenesis and fluidmigration on rare earth element distribution in pore fluids of the northern Cascadia accretionary margin. Chem. Geol. 291, 152165.

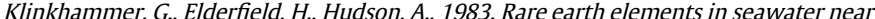
hydrothermal vents. Nature 305, 185-188.

Klinkhammer, G.P., Elderfield, H., Edmond, J.M., Mitra, A., 1994a. Geochemical implications of rare earth element patterns in hydrothermal fluids from mid-ocean ridges. Geochim. Cosmochim. Acta 58, 5105-5113.

Klinkhammer, G.P., German, C.R., Elderfield, H., Greaves, M.J., Mitra, A., 1994b. Rare earthlements in hydrothermal fluids and plume particulates by inductively coupled plasma mass spectrometry. Mar. Chem. 45, 179-186.

Klug, C., Schulz, H., De Baets, K., 2009. Red Devonian trilobites with green eyes from Morocco and the silicification of the trilobite exoskeleton. Acta Palaeontol. Pol. 54, 117-123.

Konno, U., Tsunogai, U., Nakagawa, F., Nakaseama, M., Ishibashi, J., Nunoura, T., Nakamura K., 2006. Liquid $\mathrm{CO}_{2}$ venting on the seafloor: Yonaguni Knoll IV hydrothermal system, Okinawa Trough. Geophys. Res. Lett. 33, L16607.

McLennan, S.M., 1989. Rare earth elements in sedimentary rocks: influence of provenance and sedimentary processes. In: Lipin, B.R., McKay, G.A. (Eds.), Geochemistry and Mineralogy of Rare Earth Elements. Miner. Soc. Am. Rev. Miner 21, pp. 169-200.

Michard, A., 1976. Elements de Géologie Marocaine. Notes Mém. Serv. Géol. Maroc. 252, $1-408$.

Michard, A., 1989. Rare earth element systematics in hydrothermal fluids. Geochim Cosmochim. Acta 53, 745-750.

Mitra, A., 1994. Geochemical implications of rare earth element patterns in hydrothermal

fluids from mid-ocean ridges. Geochim. Cosmochim. Acta 58, 5105-5113. Moldovanyi, E.P. Walter, L.M., Brannon, J.C., Podosek, F.A., 1990. New constraints on carbonate diagenesis from integrated $\mathrm{Sr}$ and $\mathrm{S}$ isotopic and rare earth element data, Jurassic Smackover Formation, U.S. Gulf Coast. Appl. Geochem. 5, 449-470. Monty, C.L.V.,

1995. The rise and nature of carbonate mudmounds: an introductory actualistic approach. In: Monty, C.L.V., Bosence, D.W.J., Bridges, P.H., Pratt, B.R. (Eds.), Carbonate Mud-Mounds, Their Origin and Evolution. Spec. Publ. Int. Assoc. Sedimentol. 23. Blackwell, Oxford, pp. 11-48.

Morse, J.W., Arvidson, R.S., Lüttge, A., 2007. Calcium carbonate formation and dissolution. Chem. Rev. 107, 342-381.

Mounji, D., Bourque, P.A., Savard, M.M., 1998. Hydrothermal origin of Devonian conical mounds (Kess-Kess) of Hamar Laghdad Ridge, Anti-Atlas, Morocco. Geology 26,

1123-1126.

Murray, R.W., Buchholtz Ten Brink, M.R., Gerlach, D.C., Price Russ III, G., Jones, D.L., 1991. Rare earth, major, and trace elements in chert from the Franciscan Complex and Monterey Group, California: assessing sources to fine-grained marine sediments. Geochim. Cosmochim. Acta 55, 1875-1895.

Nance, W.B., Taylor, S.R., 1976. Rare earth element patterns and crustal evolution-I. Australian post-Archean sedimentary rocks. Geochem. Cosmochem. Acta 40, 1539-1551.

Nothdurft, L.D., Webb, G.E., Kamber, B.S., 2004. Rare earth element geochemistry of Late Devonian reefal carbonates, Canning Basin, western Australia: confirmation of a seawater REE proxy in ancient limestones. Geochim. Cosmochim. Acta 68, 263-283.

Nozaki, Y., Zhang, J., Amakawa, H., 1997. The fractionation between $Y$ and Ho in the marine environment. Earth Planet. Sci. Lett. 148, 329-340.

Olempska, E., Betka, Z., 2010. Hydrothermal vent myodocopid ostracods from the Eifelian (Middle Devonian) of southern Morocco. Geobios 43, 519-529.

Olivarez, A.M., Owen, R.M., 1989. REE/Fe variations in hydrothermal sediments: implications for the REE content of seawater. Geochim. Cosmochim. Acta 53, 757-762. Oliveri, E. Neri, R., Bellanca, A., Riding, R., 2010. Carbonate stromatolites from a Messinian hypersaline setting in the Caltanissetta Basin, Sicily: petrographic evidence of micro-bial activity and related stable isotope and rare earth element signatures. Sedimentol-ogy 57, 142-161.

Olivier, N., Boyet, M., 2006. Rare earth and trace elements of microbialites in Upper Jurassic coral- and sponge-microbialite reefs. Chem. Geol. 230, 105-123.

Peckmann, J., Walliser, O.H., Riegel, W., Reitner, J., 1999. Signatures of hydrocarbon venting in a Middle Devonian carbonate mound (Hollard Mound) at the Hamar

Laghdad (Antiatlas Morocco). Facies 40, 281-296.

Peckmann, J., Little, C.T.S., Gill, F., Reitner, J., 2005. Worm tube fossils from the Hollard Mound hydrocarbon-seep deposit, Middle Devonian, Morocco: Palaeozoic seeprelated vestimentiferans? Palaeogeogr. Palaeoclimatol. Palaeoecol. 227, 242-257.

Piepgras, D.J., Jacobsen, S.B., 1992. The behavior of rare earth elements in seawater: precise determination of variations in the North Pacific water column. Geochim. Cosmochim. Acta 56, 1851-1862.

Piqué, A., Michard, A., 1989. Moroccan Hercynides: a synopsis. The Paleozoic sedimentary and tectonic evolution at the northern margin of west Africa. Am. J. Sci. 289, 286-330. Pourret, O., Davranche, M., Gruau, G., Dia, A., 2008. New insights into cerium anomalies in organic-rich alkaline waters. Chem. Geol. 251, 120-127.

Reynard, B., Lécuyer, C., Grandjeau, P., 1999. Crystal-chemical controls on rare earth element concentrations in fossil biogenic apatite and implications for

paleoenvironmental reconstructions. Chem. Geol. 155, 233-242.

Ritger, S., Carson, B., Suess, E., 1987. Methane-derived authigenic carbonates forms by subduction-induced pore-water expulsion along the Oregon/Washington margin. Geol. Soc. Am. Bull. 98, 147-156.

Rodgers, K.A., Browne, P.R.L., Buddle, T.F., Cook, K.L., Greatrex, R.A., Hampton, W.A Herdianita, N.R., Holland, G.R., Lynne, B.Y., Martin, R., Newton, Z., Pastars, D., Sannazarro, K.L., Teece, C.I.A., 2004. Silica phases in sinters and residues from geothermal fields of New Zealand. Earth-Sci. Rev. 66, 1-61.

Rongemaille, E., Bayon, G., Pierre, C., Bollinger, C., Chu, N.C., Fouquet, $Y$., Riboulot, $V$. Voisset, M., 2011. Rare earth elements in cold seep carbonates from the Niger delta. Chem. Geol. 286, 196-206.

Shields, G. Webb, G.,2004. Has the REE composition of seawater changed over geological time? Chem. Geol. 204, 103-107.

Stampfli, G.M., Borel, G.D., 2002. A plate tectonic model for the Paleozoic and Mesozoic constrained by dynamic plate boundaries and restored synthetic oceanic isochrones. Earth Planet. Sci. Lett. 196, 17-33. 
Tait, J.A., Bachtadse, V., Soffel, H., Franke, W., 1995. Paleozoic paleogeography of the circum Atlantic continents. Terra Nostra 8, 133.

Tanaka, K., Kawabe, I., 2006. REE abundances in ancient seawater inferred from marine limestone and experimental REE partition coefficients between calcite and aqueous solution. Geochem. J. 40, 425-435.

Tanaka, K., Miura, N., Asahara, Y., Kawabe, I., 2003. Rare earth element and strontium

iso- topic study of seamount-type limestones in Mesozoic accretionary complex of South-ern Chichibu Terrane, central Japan: implications for incorporation process of seawater REE into limestones. Geochem. J. 37, 163-180.

Taylor, S.R., McLennan, S.M., 1985. The Continental Crust: its Composition and Evolution. Blackwell Scientific Pub, Palo Alto, CA (328 pp.).

Tribovillard, N., Algeo, T.J., Lyons, T., Riboulleau, A., 2006. Trace metals as paleoredox and paleoproductivity proxies: an update. Chem. Geol. 232, 12-32.

Tunnicliffe, V., Juniper, S.K., Sibuet, M., 2003. Reducing environments of the deep sea floor In: Tyler, P.A. (Ed.), Ecosystems of the World: the Deep Sea. Elsevier Press,

Amsterdam, pp. 81-110.

Van Kranendonk, M.J., Webb, G.E., Kamber, B.S., 2003. Geological and trace element evidence for a marine sedimentary environment of deposition and biogenicity of 3.45 Ga stromatolitic carbonates in the Pilbara Craton, and support for a reducing Archaean ocean. Geobiology 1, 91-108.
Webb, G.E., Kamber, B.S., 2000. Rare earth elements in Holocene reefal microbialites: a new shallow seawater proxy. Geochim. Cosmochim. Acta 64, 1557-1565.

Webb, G.E., Nothdurft, L.D., Kamber, B.S., Kloprogge, J.T., Zhao, J.-X., 2009. Rare earth element geochemistry of scleractinian coral skeleton during meteoric diagenesis: a se-quence through neomorphism of aragonite to calcite. Sedimentology 56, 1433-1463. Wendt, J., 1985. Disintegration of the continental margin of northwestern Gondwana: Late Devonian of the eastern Anti-Atlas (Morocco). Geology 13, 815-818.

White, S.N., 2009. Laser Raman spectroscopy as a technique for identification of seafloor hydrothermal and cold seep minerals. Chem. Geol. 259, 240-252.

Zhang, J., Nozaki, Y., 1996. Rare earth elements and yttrium in seawater: ICP-MS determinations in the East Caroline, Coral Sea, and South Fiji basins of the western South Pacific

Ocean. Geochim. Cosmochim. Acta 60, 4631-4644.

Zhang, J., Nozaki, Y., 1998. Behavior of rare earth elements in seawater at the ocean margin: a study along the slopes of the Sagami and Nankai troughs near Japan. Geochim. Cosmochim. Acta 62, 1307-1317.

Zhang, J., Amakawa, H., Nozaki, Y., 1994. The comparative behaviors of yttrium and lanthanides in seawater of the North Pacific. Geophys. Res. Lett. 21, 2677-2680. 